\title{
RELATIVE ABELIAN KERNELS OF SOME CLASSES OF TRANSFORMATION MONOIDS
}

\author{
E. Cordeiro, M. Delgado and V.H. Fernandes
}

We consider the symmetric inverse monoid $\mathcal{I}_{n}$ of an $n$-element chain and its in-

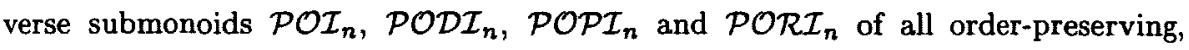
order-preserving or order-reversing, orientation-preserving and orientation-preserving or orientation-reversing transformations, respectively, and give descriptions of their Abelian kernels relative to decidable pseudovarieties of Abelian groups.

\section{INTRODUCTION}

The computability of kernels of finite monoids became a popular problem in Finite Semigroup Theory after a paper of Rhodes and Tilson [23]. Independent solutions were given by Ash [3] and Ribes and Zalesskir [24]. Both solutions contains deep results which led to the development of the theory in various directions. See for instance $[19,2]$. Computing kernels relative to other pseudovarieties had then interested various researchers; for examples, see Ribes and Zalesskir [25], the second author [5] or Steinberg [26]. As computing kernels are directly connected with the Mal'cev product of pseudovarieties of monoids where the second factor is a pseudovariety of groups (see [18]), decidability results can be obtained in this way.

The interest of the second author in using in practice the algorithm to compute the Abelian kernel of a finite monoid obtained in [5] led him to work towards a concrete implementation. Having this idea in mind, by detailing some aspects and improving the efficiency of that algorithm (see [6]) a concrete implementation in GAP [27] was then produced.

Following this, the second and third authors [7, 9] achieved fruitful computations involving classes of monoids for which extremely simple presentations were previously determined by the third author $[14,15,16]$ or by third author together with Gomes and Jesus [17].

\footnotetext{
Received 11th January, 2006

The first and second authors were (partially) supported by the Centro de Matemtica da Universidade do Porto (CMUP), financed by FCT (Portugal) through the programmes POCTI and POSI, with national and European Community structural funds. The third author was (partially) supported by FCT and FEDER whithin the project POCTI-ISFL-1-143 of the Centro de Álgebra da Universidade de Lisboa.
}

Copyright Clearance Centre, Inc. Serial-fee code: 0004-9727/06 \$A2.00+0.00. 
We observe that the interest of this kind of computations goes far beyond the computations themselves. For example, they have been at the basis of theoretical results such as those obtained by the second and third authors in [8], which extend the notion of solvability of groups, and later generalised with the collaboration of Margolis and Steinberg [10].

In this work we consider kernels relative to decidable pseudovarieties of Abelian groups. An algorithm was obtained by Steinberg [26], from which the first and second authors [4] described the necessary details to achieve an implementation using GAP. The implementation used to perform tests that led us to get a better intuition and, ultimately, to the results presented in this paper, was achieved by the second author with the collaboration of Jose Morais, using the GAP language. The GAP package SgpViz [11] has been very useful to visualise the results.

Besides this brief introduction, this paper is divided into four main sections. A fifth section containing a few consequences and conjectures is also included.

The first section contains the preliminaries and is divided into several subsections. Notation and definitions are given. Several results are recalled and some others are new and proved here. These results are to be used in Sections 2 and 4.

In Section 2 we compute the relative Abelian kernels of finite cyclic, dihedral and symmetric groups. These are the maximal subgroups of the monoids considered in this paper.

Aiming to keep the paper as self-contained as possible, we present in Section 3 a summary of the relevant results on presentations of the transformation monoids $\mathcal{P O I _ { n }}$, $\mathcal{P O D \mathcal { I } _ { n }}, \mathcal{P O P} \mathcal{I}_{n}, \mathcal{P O R} \mathcal{I}_{n}$ and $\mathcal{I}_{n}$.

In Section 4 we give descriptions of the kernels relative to all decidable pseudovarieties of Abelian groups of the transformation monoids considered in Section 3.

\section{Preliminaries}

A pseudovariety of groups is a class of finite groups closed under formation of finite direct products, subgroups and homomorphic images. In this paper we are particularly interested in the class Ab of all finite Abelian groups, which is clearly a pseudovariety, and its subpseudovarieties.

It is well known that the computation of the kernel of a finite semigroup relative to a pseudovariety of groups can be reduced to the computation of the kernel of a finite monoid relative to the same pseudovariety of groups, so we shall mainly be concerned with monoids, as is usual.

For general background on Green relations and inverse semigroups, we refer the reader to Howie's book [20]. For general notions on profinite topologies and finite semigroups, Almeida's book [1] is our reference.

This section is divided into several subsections. The first one recalls a connection 
between supernatural numbers and pseudovarieties of Abelian groups. Then we introduce some notation. The third subsection recalls an algorithm due to Steinberg [26] to compute the closure of a subgroup of a finitely generated free Abelian group relative to the profinite topology of the free Abelian group given by a decidable pseudovariety of Abelian groups. The definition of relative kernel of a finite monoid is recalled in Subsection 1.4. In Subsection 1.5 we prove two general results that will be applied in Section 2 to the groups considered in this paper. In Subsection 1.6 we prove a combinatorial result that will be used in Subsection 4.4 to describe the relative Abelian kernels of the monoids $\mathcal{P O R I}_{n}$.

1.1. Supernatural numbers and pSeudovarieties of Abelian groups. A supernatural number is a formal product of the form $\pi=\Pi_{p} p^{n_{p}}$ where $p$ runs over all natural prime numbers and $0 \leqslant n_{p} \leqslant+\infty$.

We say that a supernatural number $\Pi p^{n_{p}}$ has finite support if all $n_{p}$, except possibly a finite number, are zero. There is an obvious notion of division of supernatural numbers, which in turn leads to the notion of greatest common divisor of two supernatural numbers. A supernatural number is said to be recursive if the set of all natural numbers which divide it is recursive. In particular, supernatural numbers of finite support are recursive. A supernatural number $\Pi p^{n_{p}}$ of finite support such that all the exponents $n_{p}$ are finite is said to be finite and is naturally identified with a positive natural number. All other supernatural numbers are said to be infinite. In general we use the Greek letter $\pi$ for a (possibly infinite) supernatural number, but when the supernatural number is known to be finite, we prefer to use a roman letter, for example, $k$. We say that the supernatural number $\pi$ is odd when $\operatorname{gcd}(2, \pi)=1$ and that it is even otherwise, that is, when $\operatorname{gcd}(2, \pi)=2$.

To a supernatural number $\pi$ we associate the pseudovariety of Abelian groups $H_{\pi}$ of all finite Abelian groups whose torsion coefficients divide $\pi$ (that is, $H_{\pi}=\langle\{\mathbb{Z} / m \mathbb{Z} \mid$ $m$ divides $\pi\}\rangle)$. See Steinberg's paper [26] for details. Notice that the pseudovariety of Abelian groups associated to a natural number $k$ is just $\langle\mathbb{Z} / k \mathbb{Z}\rangle$, the pseudovariety generated by the cyclic group of order $k$. In particular, the pseudovariety of groups corresponding to the natural number 1 is the trivial pseudovariety. Observe that to the supernatural number $\Pi p^{\infty}$, where $p$ runs over all positive prime numbers, is associated the pseudovariety $\mathrm{Ab}$ of all finite Abelian groups. Observe also that decidable pseudovarieties of Abelian groups correspond to recursive supernatural numbers and that the converse is also true. All supernatural numbers considered in this paper are recursive.

For a pseudovariety $\mathrm{H}$ of groups and a finite set $A$, we denote by $F_{\mathrm{H}}(A)$ the relatively free group on $A$ in the variety of groups (in the Birkhoff sense) generated by $H$.

Let $\pi$ be a supernatural number and $H_{\pi}$ the corresponding pseudovariety of Abelian groups. The following holds:

Proposition 1.1. ([26]) Let $n$ be a positive integer and let $A$ be a finite set 
of cardinality $n$. Then if $\pi$ is a natural number, $F_{\mathrm{H}_{\pi}}(A)=(\mathbb{Z} / \pi \mathbb{Z})^{n}$. Otherwise, that is, when $\pi$ is infinite, $F_{\mathrm{H}_{\pi}}(A)=\mathbb{Z}^{n}$, the free Abelian group on $n$ generators.

It turns out that the pseudovarieties of Abelian groups corresponding to natural numbers are locally finite, while those corresponding to infinite supernatural numbers are non locally finite. The relatively free groups appearing in the last proposition will be turned into topological spaces, the finite ones being discrete.

1.2. Notation. Throughout the paper $n$ will denote a positive integer. Without surprise, after Proposition 1.1, the free Abelian group $\mathbb{Z}^{n}$ plays a fundamental role here.

In order to render our notation more understandable, we shall use subscripts in some components of the elements of $\mathbb{Z}^{n}$. For instance, we write $\left(0, \ldots, 0,1_{(i)}, 0, \ldots, 0\right)$ with the meaning of " $(0, \ldots, 0,1,0, \ldots, 0)$ ( 1 is in the position $i)$ ". We adopt the usual notation for the neutral element of an Abelian group: $(0, \ldots, 0) \in \mathbb{Z}^{n}$ is simply denoted by 0 . The set of non-negative integers, also named natural numbers, is a monoid under addition and is denoted by $\mathbb{N}$.

Let $A=\left\{a_{1}, \ldots, a_{n}\right\}$ be a finite ordered alphabet. The canonical homomorphism $\gamma: A^{*} \rightarrow \mathbb{Z}^{n}$, from the free monoid on $A$ into the $n$-generated free Abelian group, defined by $\gamma\left(a_{i}\right)=\left(0, \ldots, 0,1_{(i)}, 0, \ldots, 0\right)$ will be widely used (for the alphabets in the context). The image under $\gamma$ of a rational language of $A^{*}$ is a rational subset of $\mathbb{N}^{n}\left(\subseteq \mathbb{Z}^{n}\right)$, thus it is a semilinear set, that is, a finite union of sets of the form $a+b_{1} \mathbb{N}+\cdots+b_{r} \mathbb{N}$. There exists an algorithm (see, for instance, $[\mathbf{5}, \mathbf{6}]$ ) to compute (a semilinear expression for) the image of a rational language of $A^{*}$ by $\gamma$, when it is given by means of a rational expression.

Suppose that $M$ is an $A$-generated finite monoid and let $\varphi: A^{*} \rightarrow M$ be an onto homomorphism. We say that an element $x \in M$ can be represented by a word $w \in A^{*}$ (or that $w$ is a representation of $x$ ) if $\varphi(w)=x$.

When a monoid $M$ is given through a monoid presentation $\langle A \mid R\rangle$, we always assume that $A$ is an ordered alphabet and $\varphi$ denotes the homomorphism $\varphi: A^{*} \rightarrow M$ associated to the presentation. Thus, a presentation of a monoid determines two homomorphisms which will be represented throughout the paper by the Greek letters $\varphi$ and $\gamma$.

1.3. TOPOLOGIES FOR THE FREE ABELIAN GROUP. Let $\pi$ be an infinite supernatural number and $H_{\pi}$ the corresponding pseudovariety of Abelian groups.

The pro- $H_{\pi}$ topology on $\mathbb{Z}^{n}$ is the least topology rendering continuous all homomorphisms of $\mathbb{Z}^{n}$ into groups of $H_{\pi}$. The free Abelian group $\mathbb{Z}^{n}$ endowed with this topology is a topological group. When $H_{\pi}=A b$, the pseudovariety of all finite Abelian groups, the pro- $\mathrm{H}_{\pi}$ topology is usually referred simply as profinite topology.

For a subset $X$ of $\mathbb{Z}^{n}$, we denote by $\mathrm{Cl}_{\mathrm{H}_{\pi}}(X)$ the pro- $\mathrm{H}_{\pi}$ closure of $X$. The pro-Ab closure will in general be referred as the profinite closure. The following holds [5].

PROPOSITION 1.2. For $a, b_{1}, \ldots, b_{r} \in \mathbb{N}^{n}$, the profinite closure of the subset 
$a+b_{1} \mathbb{N}+\cdots+b_{r} \mathbb{N}$ of $\mathbb{Z}^{n}$ is $a+b_{1} \mathbb{Z}+\cdots+b_{r} \mathbb{Z}$.

Next we recall an algorithm to compute the pro- $\mathrm{H}_{\pi}$ closure of a subgroup $G$ of the free Abelian group $\mathbb{Z}^{n}$. See $[4,26]$ for details.

Let $M$ be a matrix whose rows generate $G$. We say that $M$ represents $G$. Notice that, by adding rows of zeros when necessary, we may suppose that $M$ is an $n \times n$ matrix. AlgoRITHM 1.3. INPUT: a subgroup $G$ of $\mathbb{Z}^{n}$ given through an integer $n \times n$ matrix $M$.

OUTPUT: a matrix representing the pro- $\mathrm{H}_{\pi}$ closure of $G$.

(1) Compute invertible integer matrices $P$ and $Q$ such that

$$
P M Q=S=\left[\begin{array}{ccccc}
a_{1} & 0 & 0 & \ldots & 0 \\
0 & a_{2} & 0 & \ldots & 0 \\
\vdots & \vdots & \vdots & & \vdots \\
0 & 0 & 0 & \ldots & a_{n}
\end{array}\right]
$$

is a diagonal matrix. Notice that if, for $1 \leqslant i \leqslant n-1, a_{i} \mid a_{i+1}$, then the matrix $S$ is in Smith Normal Form.

(2) For each $a_{i}$, compute $b_{i}=\operatorname{gcd}\left(a_{i}, \pi\right)$ (note that we are assuming that $\pi$ is recursive) and consider the matrix

$$
\bar{S}=\left[\begin{array}{ccccc}
b_{1} & 0 & 0 & \ldots & 0 \\
0 & b_{2} & 0 & \ldots & 0 \\
\vdots & \vdots & \vdots & & \vdots \\
0 & 0 & 0 & \ldots & b_{n}
\end{array}\right]
$$

(3) Return the matrix $\bar{S} Q^{-1}$.

The matrix returned represents the pro- $\mathrm{H}_{\pi}$ closure of $G$.

Next example illustrates the usage of the algorithm. It will be referred in Section 2.2. EXAMPLE 1.4. (1) Let $G=n \mathbb{Z}$. Then $\mathrm{Cl}_{\mathrm{H}_{\pi}}(G)=\operatorname{gcd}(n, \pi) \mathbb{Z}$. More generally, let $G$ be a subgroup of $\mathbb{Z}^{n}$ represented by a diagonal matrix. A matrix representing the pro- $\mathrm{H}_{\pi}$ closure of $G$ can be obtained from $M$ by replacing each nonzero entry $a$ with $\operatorname{gcd}(a, \pi)$.

(2) Let $G$ be the subgroup of $\mathbb{Z}^{2}$ represented by the matrix $M=\left[\begin{array}{ll}2 & 0 \\ 1 & 1\end{array}\right]$. Then

$$
\left[\begin{array}{cc}
0 & 1 \\
-1 & 2
\end{array}\right]\left[\begin{array}{cc}
2 & 0 \\
1 & 1
\end{array}\right]\left[\begin{array}{cc}
1 & -1 \\
0 & 1
\end{array}\right]=\left[\begin{array}{ll}
1 & 0 \\
0 & 2
\end{array}\right]
$$

In order to use the notation of the algorithm just stated, we write $S=\left[\begin{array}{ll}1 & 0 \\ 0 & 2\end{array}\right]$ and $Q=\left[\begin{array}{cc}1 & -1 \\ 0 & 1\end{array}\right]$. We thus have $Q^{-1}=\left[\begin{array}{cc}1 & -1 \\ 0 & 1\end{array}\right]^{-1}=\left[\begin{array}{ll}1 & 1 \\ 0 & 1\end{array}\right]$. 

If $\pi$ is even, then $\operatorname{gcd}(2, \pi)=2$. So, in this case, $\bar{S}=\left[\begin{array}{ll}1 & 0 \\ 0 & 2\end{array}\right]$ and $\mathrm{Cl}_{\mathrm{H}_{\pi}}(G)$ can be
represented by

$$
\bar{S} Q^{-1}=\left[\begin{array}{ll}
1 & 0 \\
0 & 2
\end{array}\right]\left[\begin{array}{ll}
1 & 1 \\
0 & 1
\end{array}\right]=\left[\begin{array}{ll}
1 & 1 \\
0 & 2
\end{array}\right]
$$

and therefore $\mathrm{Cl}_{\mathrm{H}_{\pi}}(G)=G$.

If $\pi$ is odd, then $\operatorname{gcd}(2, \pi)=1$ and we get $\bar{S} Q^{-1}=\left[\begin{array}{ll}1 & 1 \\ 0 & 1\end{array}\right]$. Thus $\{(1,1),(0,1)\}$ is a basis of the pro- $\mathrm{H}_{\pi}$ closure of $G$ and it follows that $\mathrm{Cl}_{H_{\pi}}(G)=\mathbb{Z}^{2}$.

1.4. Relative KeRNels of FINITE MONOIDS. Let $M$ and $N$ be finite monoids. A relational morphism of monoids $\tau: M \rightarrow N$ is a function from $M$ into $\mathcal{P}(N)$, the power set of $N$, such that:

(a) For all $s \in M, \tau(s) \neq \emptyset$;

(b) For all $s_{1}, s_{2} \in M, \tau\left(s_{1}\right) \tau\left(s_{2}\right) \subseteq \tau\left(s_{1} s_{2}\right)$;

(c) $1 \in \tau(1)$.

A relational morphism $\tau: M \rightarrow N$ is, in particular, a relation in $M \times N$. Thus, composition of relational morphisms is naturally defined. Homomorphisms, seen as relations, and inverses of onto homomorphisms are examples of relational morphisms.

Given a pseudovariety $\mathrm{H}$ of groups, the $\mathrm{H}$-kernel of a finite monoid $M$ is the submonoid $\mathrm{K}_{\mathrm{H}}(M)=\cap \tau^{-1}(1)$, with the intersection being taken over all groups $G \in \mathrm{H}$ and all relational morphisms of monoids $\tau: M \rightarrow G$. Sometimes we refer to the H-kernel simply as relative kernel. When $\mathrm{H}$ is $\mathrm{Ab}$, we use the terminology Abelian kernel. Accordingly, when $\mathrm{H}$ is a subpseudovariety of $\mathrm{Ab}$, we say relative Abelian kernel.

As an example we can state the following proposition which was proved in a slightly more general form in [5]. See also [7].

PROPOSITION 1.5. The Abelian kernel of a finite group is precisely its derived subgroup.

All statements of the following remark follow directly from the definition or are easy to prove (and well known, in any case).

REMARK 1.6. Let $\mathrm{H}$ and $\mathrm{H}^{\prime}$ be pseudovarieties of groups and let $M$ be a finite monoid. Then

(1) If $\mathrm{H} \subseteq \mathrm{H}^{\prime}$, then $\mathrm{K}_{\mathrm{H}^{\prime}}(M) \subseteq \mathrm{K}_{\mathrm{H}}(M)$.

(2) If $N$ is a subsemigroup of $M$ that is a monoid, then $\mathrm{K}_{\mathrm{H}}(N) \subseteq \mathrm{K}_{\mathrm{H}}(M) \cap N$.

(3) $\mathrm{K}_{\mathrm{H}}(M)$ contains the idempotents of $M$.

When the subsemigroup of $M$ mentioned in the second statement of previous remark is the group of units, the inclusion can be replaced by an equality, as stated in the following lemma. 
LEMma 1.7. ([7, Lemma 4.8]) Let $G$ be the group of units of a finite monoid $M$. Then $\mathrm{K}_{\mathrm{H}}(M) \cap G=\mathrm{K}_{\mathrm{H}}(G)$, for any pseudovariety of groups $\mathrm{H}$. In particular, $\mathrm{K}_{\mathrm{Ab}}(M) \cap G=G^{\prime}$.

As a consequence of Proposition 1.5 and the first step of Remark 1.6 we get the following.

COROLlary 1.8. Any relative Abelian kernel of a finite group contains its derived subgroup.

Let $\pi$ be an even supernatural number. It is clear that $\mathrm{H}_{2} \subseteq \mathrm{H}_{\pi}$, thus, for any finite monoid $M, \mathrm{~K}_{\mathrm{Ab}}(M) \subseteq \mathrm{K}_{\mathrm{H}_{\pi}}(M) \subseteq \mathrm{K}_{\mathrm{H}_{2}}(M)$. Consequently we have the following.

Proposition 1.9. Let $M$ be a finite monoid such that $\mathrm{K}_{\mathrm{H}_{2}}(M)=\mathrm{K}_{\mathrm{Ab}}(M)$. Then, for any even supernatural number $\pi, \mathrm{K}_{\mathrm{H}_{\pi}}(M)=\mathrm{K}_{\mathrm{Ab}}(M)$.

1.5. SOME RESULTS CONCERNING RELATIVE ABELIAN KERNELS. Let $\pi$ be an infinite supernatural number, let $H_{\pi}$ be the corresponding pseudovariety of Abelian groups and let $M$ be a finite $A$-generated monoid. The following result was proved by the second author [5] for the case $\mathrm{H}_{\pi}=A b$ and generalised by Steinberg [26] to cover all other cases.

Proposition 1.10. Let $x \in M$. Then $x \in \mathrm{K}_{\mathrm{H}_{\pi}}(M)$ if and only if $0 \in \mathrm{Cl}_{H_{\pi}}\left(\gamma\left(\varphi^{-1}(x)\right)\right)$.

Next we recall some facts proved by the first and second authors [4]. In order to compute $\mathrm{Cl}_{\mathrm{H}_{\pi}}\left(\gamma\left(\varphi^{-1}(x)\right)\right)$ we can calculate $\mathrm{Cl}_{\mathrm{Ab}}\left(\gamma\left(\varphi^{-1}(x)\right)\right)$ in an intermediate step, as the next result shows.

Proposition 1.11. Let $x \in M$. Then

$$
\mathrm{Cl}_{\mathrm{H}_{\pi}}\left(\gamma\left(\varphi^{-1}(x)\right)\right)=\mathrm{Cl}_{\mathrm{H}_{\pi}}\left(\mathrm{Cl}_{\mathrm{Ab}}\left(\gamma\left(\varphi^{-1}(x)\right)\right)\right) .
$$

Now let $k$ be a finite supernatural number and $H_{k}$ be the corresponding pseudovariety of Abelian groups. We consider the projection $c_{k}: \mathbb{Z}^{n} \rightarrow(\mathbb{Z} / k \mathbb{Z})^{n}$ (defined by: $\left.c_{k}\left(r_{1}, \ldots, r_{n}\right)=\left(r_{1} \bmod k, \ldots, r_{n} \bmod k\right)\right)$ and the homomorphism $\gamma_{k}=c_{k} \circ \gamma: A^{*}$ $\rightarrow(\mathbb{Z} / k \mathbb{Z})^{n}$. Note that for a word $w \in A^{*}$, the $i^{\text {th }}$ component of $\gamma_{k}(w)$ is the number of occurrences modulo $k$ of the $i^{\text {th }}$ letter of $A$ in $w$.

The next proposition is similar to Proposition 1.11. It allows us to compute $\gamma_{k}\left(\varphi^{-1}(x)\right)$ using $\mathrm{Cl}_{\mathrm{Ab}}\left(\gamma\left(\varphi^{-1}(x)\right)\right)$ in an intermediate step.

PROPOSITION 1.12. Let $x \in M$. Then $\gamma_{k}\left(\varphi^{-1}(x)\right)=c_{k}\left(\mathrm{Cl}_{\mathrm{Ab}}\left(\gamma\left(\varphi^{-1}(x)\right)\right)\right)$.

An analog to Proposition 1.10 was also stated in [4].

Proposition 1.13. Let $x \in M$. Then $x \in \mathrm{K}_{\mathrm{H}_{\mathrm{k}}}(M)$ if and only if $0 \in \gamma_{k}\left(\varphi^{-1}(x)\right)$.

The following is another simple and useful characterisation of the $\mathrm{H}_{k}$-kernel proved in the same paper. 
PROPOSITION 1.14. Let $x \in M$. Then $x \in \mathrm{K}_{\mathrm{H}_{k}}(M)$ if and only if $x$ can be represented by a word $w \in A^{*}$ such that, for any letter $a \in A,|w|_{a} \equiv 0 \bmod k$.

Note that, as a consequence, we get that if $x \in \mathrm{K}_{\mathrm{H}_{k}}(M)$, then $x$ can be represented by a word whose length is a multiple of $k$. The following result and its proof is similar to $[9$, Theorem 3.4].

THEOREM 1.15. Let $T$ be a monoid, let $x_{1}, x_{2}, \ldots, x_{s}, y$ be a set of generators of $T$ such that $y^{k}=1$ and let $S$ be the submonoid of $T$ generated by $x_{1}, x_{2}, \ldots, x_{s}$. If for each $i \in\{1, \ldots, s\}$ there exists $u_{i} \in S$ such that $y x_{i}=u_{i} y$, then $\mathrm{K}_{\mathrm{H}_{k}}(T) \subseteq S$.

ProOF: Let $x \in \mathrm{K}_{\mathrm{H}_{\mathrm{k}}}(T)$. Then, by Proposition 1.14, we can represent $x$ as a word $w \in\left\{x_{1}, x_{2}, \ldots, x_{s}, y\right\}^{*}$ with a number of occurrences of the generator $y$ that is a multiple of $k$. Then, applying the relations $y x_{i}=u_{i} y, i \in\{1, \ldots, s\}$ from the left hand side to the right hand side of $w$ and the relation $y^{k}=1$ whenever possible, we can represent $x$ without any occurrence of $y$, thus $x \in S$.

Another important result of this subsection is Proposition 1.17 that gives a simple and efficient way to compute the $\mathrm{H}_{\mathrm{k}}$-kernel of a finite group. In order to state it, we need to introduce a subgroup of a group containing the derived subgroup.

Given a finite group $G$, denote by $G^{[k]}$ the subgroup of $G$ generated by the commutators of $G$ (that is, the elements of the form $x y x^{-1} y^{-1}, x, y \in G$ ) and by the $k$-powers of $G$ (that is, the elements of the form $x^{k}, x \in G$ ). In other words, $G^{[k]}$ is the smallest subgroup of $G$ containing the derived subgroup $G^{\prime}$ and the $k$-powers.

LEMMA 1.16. The subgroup $G^{[k]}$ is normal in $G$.

Proof: Let $G_{1}$ and $G_{2}$ be finite groups and let $\varphi: G_{1} \rightarrow G_{2}$ be a homomorphism. We have that

$$
\varphi\left(x y x^{-1} y^{-1}\right)=\varphi(x) \varphi(y) \varphi\left(x^{-1}\right) \varphi\left(y^{-1}\right)=\varphi(x) \varphi(y)(\varphi(x))^{-1}(\varphi(y))^{-1}
$$

and $\varphi\left(x^{k}\right)=(\varphi(x))^{k}$, thus $\varphi\left(G_{1}{ }^{[k]}\right) \subseteq G_{2}{ }^{[k]}$.

In particular, taking the inner automorphism $\varphi_{g}: G \rightarrow G$ defined by $\varphi_{g}(x)=g x g^{-1}$, we have that, for any $g \in G, g G^{[k]} g^{-1} \subseteq G^{[k]}$, concluding the proof.

Since $G^{\prime} \subseteq G^{[k]}$, we have that the factor group $G / G^{[k]}$ is Abelian. Furthermore, the order of any element $x G^{[k]}$ of $G / G^{[k]}$ divides $k$, which implies that $G / G^{[k]} \in \mathrm{H}_{\mathrm{k}}$.

PRopositION 1.17. For a finite group $G$, we have: $\mathrm{K}_{\mathrm{H}_{\mathrm{k}}}(G)=G^{[k]}$.

PROOF: The projection $p: G \rightarrow G / G^{[k]}$ is such that $p^{-1}(1)=G^{(k)}$, thus $\mathrm{K}_{\mathrm{H}_{k}}(G)$ $\subseteq G^{[k]}$.

Conversely, by Corollary 1.8, $G^{\prime} \subseteq \mathrm{K}_{\mathrm{H}_{k}}(G)$. Since the elements of the form $x^{k}$ may be written involving each generator of $G$ a multiple of $k$ times, the result follows from Proposition 1.14 . 
1.6. A COMbinatorial Result. We end the section of preliminaries with a combinatorial result (Proposition 1.22) to be used in Section 4.3.

Let $j$ be an integer. Consider the set

$$
B(j)=\{r(n-1)-i+j \mid(i, r) \in\{1, \ldots, n-1\} \times\{0, \ldots, n-1\}\} .
$$

LEMMA $1.18 . \quad B(j)$ consists of $n(n-1)$ consecutive integers.

PROOF: Denote by $m$ and $M$, respectively, the minimum and the maximum of $B(j)$. Clearly $m=0(n-1)-(n-1)+j$ and $M=(n-1)(n-1)-1+j$. As $M-m+1=n(n-1)$, it suffices to prove $B(j)$ has cardinality $n(n-1)$ to conclude that all integers between $m$ and $M$ belong to $B(j)$.

On the other hand, as $n(n-1)$ is precisely the cardinality of $\{1, \ldots, n-1\}$ $\times\{0, \ldots, n-1\}$, to prove that $B(j)$ has also cardinality $n(n-1)$ it is enough to observe that, for $(i, r),\left(i^{\prime}, r^{\prime}\right) \in\{1, \ldots, n-1\} \times\{0, \ldots, n-1\}$, if $r(n-1)-i+j=r^{\prime}(n-1)-i^{\prime}+j$, then $i=i^{\prime}$ and $r=r^{\prime}$. Now observe that $-(n-1)<i-i^{\prime}<n-1$ and $r(n-1)-i+j$ $=r^{\prime}(n-1)-i^{\prime}+j \Leftrightarrow\left(r-r^{\prime}\right)(n-1)-\left(i-i^{\prime}\right)=0$. But this implies that $r=r^{\prime}$, from what follows that also $i=i^{\prime}$.

As an immediate consequence of the preceding lemma we get the following corollary.

Corollary 1.19. If $d$ is a divisor of $n(n-1)$, then $B(j)$ contains $n(n-1) / d$ multiples of $d$.

The proof of Lemma 1.18 has also the following corollary as an immediate consequence.

Corollary 1.20. The function $g:\{1, \ldots, n-1\} \times\{0, \ldots, n-1\} \rightarrow B(j)$ defined by $g(i, r)=r(n-1)-i+j$ is a bijection.

Let $X=\{1, \ldots, n-1\} \times\{1, \ldots, n\} \times\{0, \ldots, n-1\}$ and suppose now that $1 \leqslant j \leqslant n$. Denote by $\mathcal{U}=\bigcup_{j \in\{1, \ldots, n\}} B(j) \times\{j\}$ the disjoint union of the $B(j)$ 's. From the preceding corollary we get immediately the following.

Corollary 1.21. The function $f: X \rightarrow \mathcal{U}$ defined by $f(i, j, r)=(r(n-1)$ $-i+j, j)$ is a bijection.

Denote by $A(n, d)$ the set of elements of $X$ corresponding, via the bijection of the preceding corollary, to elements of $\mathcal{U}$ whose first component is a multiple of $d$, that is

$$
A(n, d)=\{(i, j, r) \in X: d \mid(r(n-1)+j-i)\}
$$

As a consequence of Corollary 1.19 we get the main result of this section.

PROPOSITION 1.22. If $d$ is a divisor of $n(n-1)$, then $|A(n, d)|=n^{2}(n-1) / d$. 


\section{Relative Abelian KERNELS OF SOME Finite GRoups}

As already observed, in this paper, cyclic, dihedral and symmetric groups appear frequently. This section provides the computations of relative Abelian kernels of finite groups of these kinds. We shall consider them as given by the monoid presentations of the following example. In this section $n$ is a positive integer.

EXAMPLE 2.1.

1. $C_{n}=\left\langle g \mid g^{n}=1\right\rangle$ (the cyclic group of order $n$ );

2. $D_{2 n}=\left\langle h, g \mid h^{2}=g^{n}=h g^{n-1} h g^{n-1}=1\right\rangle$ (the dihedral group of order $2 n$ );

3. $\mathcal{S}_{n}=\langle a, g| a^{2}=g^{n}=(g a)^{n-1}=\left(a g^{n-1} a g\right)^{3}=\left(a g^{n-j} a g^{j}\right)^{2}=1 \quad(2 \leqslant j$ $\leqslant n-2)\rangle$ (the symmetric group on a base set with $n$ elements).

The presentation given for $\mathcal{S}_{n}$ requires some explanation. Consider the transposition $a=(12)$ and the $n$-cycle $g=(12 \cdots n)$ of $\mathcal{S}_{n}$. Then, it is well known that $\{a, g\}$ is a set of generators of $\mathcal{S}_{n}$ and, from a group presentation due to Moore [21], one can easily deduce [16] the monoid presentation for $\mathcal{S}_{n}$ given in the above example.

As Proposition 1.10 (together with Proposition 1.11 or even Proposition 1.12) makes clear, one possible strategy to compute relative Abelian kernels is to start computing profinite closures. This is what we do in this section for the cases of kernels relative to pseudovarieties corresponding to infinite supernatural numbers.

2.1. Profinite closures. In this subsection the $n$-generated free Abelian group $\mathbb{Z}^{n}$ is considered endowed with the profinite topology.

Let $G=\left\langle A \mid r_{1}=r_{2}=\cdots=r_{s}=1\right\rangle$ be a monoid presentation of a finite group. As any word in $\varphi^{-1}(1)$ can be obtained from the empty word by inserting or removing some relators, we have that $\gamma\left(r_{1}\right) \mathbb{N}+\cdots+\gamma\left(r_{s}\right) \mathbb{N} \subseteq \gamma\left(\varphi^{-1}(1)\right) \subseteq \gamma\left(r_{1}\right) \mathbb{Z}+\cdots+\gamma\left(r_{s}\right) \mathbb{Z}$. Using Proposition 1.2 we have the following lemma.

LEMMA 2.2. With the above notation, $\mathrm{Cl}_{\mathrm{Ab}}\left(\gamma\left(\varphi^{-1}(1)\right)\right)=\gamma\left(r_{1}\right) \mathbb{Z}+\cdots+\gamma\left(r_{s}\right) \mathbb{Z}$, that is, $\mathrm{Cl}_{\mathrm{Ab}}\left(\gamma\left(\varphi^{-1}(1)\right)\right)$ is the subgroup of $\mathbb{Z}^{n}$ generated by $\gamma\left(r_{1}\right), \gamma\left(r_{2}\right), \ldots, \gamma\left(r_{s}\right)$.

Next result shows, in particular, that to compute $\mathrm{Cl}_{\mathrm{Ab}}\left(\gamma\left(\varphi^{-1}(x)\right)\right)$, where $x$ is an element of the group $G$, it is not important which representative of $x$ is used.

LEмма 2.3. Let $w_{x} \in A^{*}$ be a representative of $x \in G$. Then

$$
\mathrm{Cl}_{\mathrm{Ab}}\left(\gamma\left(\varphi^{-1}(x)\right)\right)=\gamma\left(w_{x}\right)+\mathrm{Cl}_{\mathrm{Ab}}\left(\gamma\left(\varphi^{-1}(1)\right)\right)
$$

Proof: Observe that

$$
\gamma\left(w_{x}\right)+\mathrm{Cl}_{\mathrm{Ab}}\left(\gamma\left(\varphi^{-1}(1)\right)\right)=\mathrm{Cl}_{\mathrm{Ab}}\left(\gamma\left(w_{x}\right)+\gamma\left(\varphi^{-1}(1)\right)\right),
$$

since addition in $\mathbb{Z}^{n}$ is continuous. As $w_{x} \varphi^{-1}(1) \subseteq \varphi^{-1}(x)$, we have that

$$
\gamma\left(w_{x}\right)+\gamma\left(\varphi^{-1}(1)\right) \subseteq \gamma\left(\varphi^{-1}(x)\right),
$$


which implies that

$$
\gamma\left(w_{x}\right)+\mathrm{Cl}_{\mathrm{Ab}}\left(\gamma\left(\varphi^{-1}(1)\right)\right) \subseteq \mathrm{Cl}_{\mathrm{Ab}}\left(\gamma\left(\varphi^{-1}(x)\right)\right) .
$$

For the reverse inclusion it suffices to observe that

$$
\gamma\left(\varphi^{-1}(x)\right) \subseteq \gamma\left(w_{x}\right)+\mathrm{Cl}_{\mathrm{Ab}}\left(\gamma\left(\varphi^{-1}(1)\right)\right) .
$$

But this is immediate, since any word $w$ representing $x$ can be obtained from $w_{x}$ by inserting or removing the relators a finite number of times, thus

$$
\gamma(w) \in \gamma\left(w_{x}\right)+\gamma\left(r_{1}\right) \mathbb{Z}+\cdots+\gamma\left(r_{s}\right) \mathbb{Z}=\gamma\left(w_{x}\right)+\mathrm{Cl}_{\mathrm{Ab}}\left(\gamma\left(\varphi^{-1}(1)\right)\right) .
$$

Next we apply the results just obtained to the groups $C_{n}, D_{2 n}$ and $\mathcal{S}_{n}$.

THE CASE OF THE CYCLIC GROUP $C_{n}$. Let $x \in C_{n}$ and let $r$ be a non negative integer such that $g^{r}$ is a word representing $x$ (that is, $x=\varphi\left(g^{r}\right)$ ). By Lemma 2.2 we have $\mathrm{Cl}_{\mathrm{Ab}}\left(\gamma\left(\varphi^{-1}(1)\right)\right)=n \mathbb{Z}$ and then Lemma 2.3 shows that

$$
\mathrm{Cl}_{\mathrm{Ab}}\left(\gamma\left(\varphi^{-1}(x)\right)\right)=\gamma\left(g^{r}\right)+n \mathbb{Z}=r+n \mathbb{Z} .
$$

THE CASE OF THE DIHEDRAL GROUP $D_{2 n}$. Let $x \in D_{2 n}$ and let $w_{x} \in A^{*}$ be a representative of $x$. By Lemma 2.2,

$$
\mathrm{Cl}_{\mathrm{Ab}}\left(\gamma\left(\varphi^{-1}(1)\right)\right)=(2,0) \mathbb{Z}+(0, n) \mathbb{Z}+(2,2 n-2) \mathbb{Z} .
$$

Using now Lemma 2.3 we have that

$$
\begin{aligned}
\mathrm{Cl}_{\mathrm{Ab}}\left(\gamma\left(\varphi^{-1}(x)\right)\right) & =\gamma\left(w_{\boldsymbol{x}}\right)+(2,0) \mathbb{Z}+(0, n) \mathbb{Z}+(2,2 n-2) \mathbb{Z} \\
& =\gamma\left(w_{x}\right)+(2,0) \mathbb{Z}+(0, n) \mathbb{Z}+(0,-2) \mathbb{Z} \\
& = \begin{cases}\gamma\left(w_{\boldsymbol{x}}\right)+(2,0) \mathbb{Z}+(0,1) \mathbb{Z} \text { if } n \text { is odd } \\
\gamma\left(w_{\boldsymbol{x}}\right)+(2,0) \mathbb{Z}+(0,2) \mathbb{Z} \text { if } n \text { is even. }\end{cases}
\end{aligned}
$$

THE CASE OF THE SYMMETRIC GROUP $\mathcal{S}_{n}$. Using similar notation and arguments we have

$$
\begin{aligned}
\mathrm{Cl}_{\mathrm{Ab}}\left(\gamma\left(\varphi^{-1}(x)\right)\right) & =\gamma\left(w_{x}\right)+(2,0) \mathbb{Z}+(0, n) \mathbb{Z}+(n-1, n-1) \mathbb{Z}+(6,3 n) \mathbb{Z}+(4,2 n) \mathbb{Z} \\
& =\gamma\left(w_{x}\right)+(2,0) \mathbb{Z}+(0, n) \mathbb{Z}+(n-1, n-1) \mathbb{Z} \\
& = \begin{cases}\gamma\left(w_{x}\right)+(2,0) \mathbb{Z}+(0, n) \mathbb{Z}+(0, n-1) \mathbb{Z} \text { if } n \text { is odd } \\
\gamma\left(w_{x}\right)+(2,0) \mathbb{Z}+(0, n) \mathbb{Z}+(1,1) \mathbb{Z} \quad \text { if } n \text { is even }\end{cases} \\
& = \begin{cases}\gamma\left(w_{x}\right)+(2,0) \mathbb{Z}+(0,1) \mathbb{Z} \text { if } n \text { is odd } \\
\gamma\left(w_{x}\right)+(2,0) \mathbb{Z}+(1,1) \mathbb{Z} \text { if } n \text { is even. }\end{cases}
\end{aligned}
$$


2.2. Relative A belian Kernels. Next we compute relative Abelian kernels of cyclic, dihedral and symmetric groups.

We start by applying to these particular cases the fact that the Abelian kernel of a finite group is its derived subgroup (Proposition 1.5). One can easily compute the derived subgroup of a dihedral group (see [12]) and it is well known that the derived subgroup of the symmetric group is the alternating subgroup. We thus have the following lemma.

LEMMA 2.4 .

(1) The Abelian kernel of a finite Abelian group is the trivial subgroup;

(2) The Abelian kernel of the dihedral group $D_{2 n}$ of order $2 n$ is the subgroup

$$
\left\langle g^{2}\right\rangle= \begin{cases}\langle g\rangle & \text { if } n \text { is odd } \\ \left\{g^{2 i} \mid 0 \leqslant i \leqslant \frac{n}{2}\right\} & \text { if } n \text { is even }\end{cases}
$$

of $D_{2 n}$, where $g$ is the generator of order $n$;

(3) The Abelian kernel of $\mathcal{S}_{n}$ is the alternating subgroup $\mathcal{A}_{n}$.

For kernels relative to proper subpseudovarieties of $A b$ we shall distinguish the cases of pseudovarieties corresponding to infinite supernatural numbers and those corresponding to natural numbers.

Let $\pi$ be an infinite supernatural number and let $\mathrm{H}_{\pi}$ be the corresponding pseudovariety of Abelian groups. Recall (Proposition 1.10) that for $x \in G, x \in \mathrm{K}_{\mathrm{H}_{\pi}}(G)$ if and only if $0 \in \mathrm{Cl}_{\mathrm{H}_{\pi}}\left(\gamma\left(\varphi^{-1}(x)\right)\right)$. Note also that Proposition 1.11 allows us to use the computations of Subsection 2.1 to calculate $\mathrm{Cl}_{\mathrm{H}_{\pi}}\left(\gamma\left(\varphi^{-1}(x)\right)\right)$.

The CASE OF THE CYCliC Group $C_{n}$. Let $x=\varphi\left(g^{r}\right) \in C_{n}$ and let $d=\operatorname{gcd}(n, \pi)$. By the Example 1.4 we have $\mathrm{Cl}_{H_{\pi}}\left(\gamma\left(\varphi^{-1}(x)\right)\right)=r+d \mathbb{Z}$.

Since there exists $t \in \mathbb{Z}$ such that $0=r+d t$ if and only if $r$ is a multiple of $d$, we have

$$
\mathrm{K}_{\mathrm{H}_{\pi}}\left(C_{n}\right)=\left\langle g^{d}\right\rangle,
$$

where $d=\operatorname{gcd}(n, \pi)$.

THE CASE OF THE DiHedral GROUP $D_{2 n}$. Let $x \in D_{2 n}$. Then

$$
\begin{aligned}
\mathrm{Cl}_{\mathrm{H}_{\pi}}\left(\gamma\left(\varphi^{-1}(x)\right)\right) & =\mathrm{Cl}_{\mathrm{H}_{\pi}}\left(\mathrm{Cl}_{\mathrm{Ab}}\left(\gamma\left(\varphi^{-1}(x)\right)\right)\right) \\
& = \begin{cases}\mathrm{Cl}_{\mathrm{H}_{\pi}}\left(\gamma\left(w_{x}\right)+(2,0) \mathbb{Z}+(0,1) \mathbb{Z}\right) & \text { if } n \text { is odd } \\
\mathrm{Cl}_{\mathrm{H}_{\pi}}\left(\gamma\left(w_{x}\right)+(2,0) \mathbb{Z}+(0,2) \mathbb{Z}\right) & \text { if } n \text { is even. }\end{cases}
\end{aligned}
$$

We shall consider two sub-cases according to whether $\pi$ is even or $\pi$ is odd.

Subcase 1. $\pi$ even. As $\operatorname{gcd}(2, \pi)=2$, by making use of Example 1.4, we have that

$$
\mathrm{Cl}_{\mathrm{H}_{\pi}}\left(\gamma\left(\varphi^{-1}(x)\right)\right)=\left\{\begin{array}{ll}
\gamma\left(w_{x}\right)+(2,0) \mathbb{Z}+(0,1) \mathbb{Z} & \text { if } n \text { is odd } \\
\gamma\left(w_{x}\right)+(2,0) \mathbb{Z}+(0,2) \mathbb{Z} & \text { if } n \text { is even }
\end{array}=\mathrm{Cl}_{A b}\left(\gamma\left(\varphi^{-1}(x)\right)\right) .\right.
$$


Therefore

$$
\mathrm{K}_{\mathrm{H}_{\pi}}\left(D_{2 n}\right)=\mathrm{K}_{\mathrm{Ab}}\left(D_{2 n}\right)=D_{2 n}^{\prime}=\left\langle g^{2}\right\rangle .
$$

Subcase 2. $\pi$ odd. As $\operatorname{gcd}(2, \pi)=1$, by making again use of Example 1.4, we have $\mathrm{Cl}_{\mathrm{H}_{\pi}}\left(\gamma\left(\varphi^{-1}(x)\right)\right)=\mathbb{Z}^{2}$. Thus

$$
\mathrm{K}_{\mathrm{H}_{\pi}}\left(D_{2 n}\right)=D_{2 n}
$$

THE CASE OF THE SYMMETRIC GROUP $\mathcal{S}_{n}$. Let $x \in \mathcal{S}_{n}$. Then

$$
\mathrm{Cl}_{\mathrm{H}_{\pi}}\left(\gamma\left(\varphi^{-1}(x)\right)\right)= \begin{cases}\mathrm{Cl}_{\mathrm{H}_{\pi}}\left(\gamma\left(w_{x}\right)+(2,0) \mathbb{Z}+(0,1) \mathbb{Z}\right) & \text { if } n \text { is odd } \\ \mathrm{Cl}_{\mathrm{H}_{\pi}}\left(\gamma\left(w_{x}\right)+(2,0) \mathbb{Z}+(1,1) \mathbb{Z}\right) & \text { if } n \text { is even. }\end{cases}
$$

We consider again two subcases and make use of Example 1.4.

Subcase 1. $\pi$ even. We have

$$
\mathrm{Cl}_{H_{\pi}}\left(\gamma\left(\varphi^{-1}(x)\right)\right)= \begin{cases}\gamma\left(w_{x}\right)+(2,0) \mathbb{Z}+(0,1) \mathbb{Z} & \text { if } n \text { is odd } \\ \gamma\left(w_{x}\right)+(0,2) \mathbb{Z}+(1,1) \mathbb{Z} & \text { if } n \text { is even }\end{cases}
$$

thus, also in this case, $\mathrm{Cl}_{\mathrm{H}_{\pi}}\left(\gamma\left(\varphi^{-1}(x)\right)\right)=\mathrm{Cl}_{\mathrm{Ab}}\left(\gamma\left(\varphi^{-1}(x)\right)\right)$ and therefore,

$$
\mathrm{K}_{\mathrm{H}_{\pi}}\left(\mathcal{S}_{n}\right)=\mathcal{A}_{n} .
$$

Subcase 2. $\pi$ odd. Again $\mathrm{Cl}_{\mathrm{H}_{\pi}}\left(\gamma\left(\varphi^{-1}(x)\right)\right)=\mathbb{Z}^{2}$ and therefore

$$
\mathrm{K}_{\mathrm{H}_{\pi}}\left(\mathcal{S}_{n}\right)=\mathcal{S}_{n}
$$

Similar results for finite supernatural numbers could be attained in an entirely analogous way, but here we prefer to observe that these correspond to particular cases of Proposition 1.17 and give alternative proofs. Let $k$ be a natural number and let $H_{k}$ be the corresponding pseudovariety of Abelian groups.

THE CASE OF THE CYClic group $C_{n}$. Let $G=C_{n}$. Since $C_{n}$ is Abelian, there is no need to consider the commutators. Let $d=\operatorname{gcd}(n, k)$. As $d \mid k$, we have that $G^{[k]}$ $=\left\langle g^{k}\right\rangle \subseteq\left\langle g^{d}\right\rangle$. Let $r$ and $s$ be integers such that $d=r k+s n$. Thus $g^{d}=g^{r k} g^{s n}=g^{r k}$ $\in G^{[k]}$. Thus $\left\langle g^{d}\right\rangle \subseteq G^{[k]}$, and therefore $G^{[k]}=\left\langle g^{d}\right\rangle$.

The CASE OF THE DiHedral group $D_{2 n}$. Let $G=D_{2 n}$. As $\left\langle g^{2}\right\rangle=G^{\prime}$, we have that $\left\langle g^{2}\right\rangle \subseteq G^{[k]}$. Note that the relation $h g^{n-1}=g h$ follows from the defining relations for $D_{2 n}$ and therefore the elements of $D_{2 n}$ may be written in the form $g^{i}$ or $h g^{i}$, with $i \in\{1, \ldots, n\}$. We consider again two subcases, according to whether $k$ is even or odd.

Subcase 1. $\quad k$ even. As $h g h g=h^{2} g^{n-1} g=h^{2} g^{n}=1$, we may conclude that, for $i \in\{1, \ldots, n\},\left(h g^{i}\right)^{2}=h g^{i} h g^{i}=h g^{i-1} h g^{n-1} g^{i}=h g^{i-1} h g^{i-1}=1$. It follows that $G^{[k]}=\left\langle g^{2}\right\rangle=G^{\prime}$. 
Subcase 2. $k$ odd. Note that, as $k=2 r+1$, for some $r,\left(h g^{i}\right)^{k}=\left(h g^{i}\right)^{2 r+1}=h g^{i}$, for $i \in\{1, \ldots, n\}$. In particular, $h, h g \in G^{[k]}$, thus $g=h \cdot h g \in G^{[k]}$. It follows that $G^{[k]}=G$.

THE CASE OF THE SYMMETRIC GROUP $\mathcal{S}_{n}$. Let $\sigma \in \mathcal{S}_{n}$. Once again, we consider two subcases, according to whether $k$ is even or odd.

Subcase 1. $k$ even. It is clear that $\sigma^{k} \in \mathcal{A}_{n}$ and therefore $\mathcal{S}_{n}^{[k]}=\mathcal{A}_{n}=\mathcal{S}_{n}^{\prime}$.

Subcase 2. $k$ odd. In this case, $\sigma^{k}$ has the same parity than $\sigma$, thus $\mathcal{S}_{n}{ }^{[k]}$ cannot be contained in $\mathcal{A}_{n}$. Since $\mathcal{A}_{n}$ has index 2 in $\mathcal{S}_{n}, \mathcal{S}_{n}^{[k]}$ must be $\mathcal{S}_{n}$.

Summarising, we have:

THEOREM 2.5. Let $\pi$ be a (finite or infinite) supernatural number and let $\mathrm{H}_{\pi}$ be the corresponding pseudovariety of Abelian groups. Then:

(1) $\mathrm{K}_{\mathrm{H}_{\pi}}\left(C_{n}\right)=\left\langle g^{d}\right\rangle$, where $d=\operatorname{gcd}(n, \pi)$.

(2) If $\pi$ is even, then $\mathrm{K}_{\mathrm{H}_{\pi}}\left(D_{2 n}\right)=\mathrm{K}_{\mathrm{Ab}}\left(D_{2 n}\right)=D_{2 n}^{\prime}=\left\langle g^{2}\right\rangle$.

If $\pi$ is odd, then $\mathrm{K}_{\mathrm{H}_{\pi}}\left(D_{2 n}\right)=D_{2 n}$.

(3) If $\pi$ is even, $\mathrm{K}_{\mathrm{H}_{\pi}}\left(\mathcal{S}_{n}\right)=\mathrm{K}_{\mathrm{Ab}}\left(\mathcal{S}_{n}\right)=\mathcal{A}_{n}$.

If $\pi$ is odd, $\mathrm{K}_{\mathrm{H}_{\pi}}\left(\mathcal{S}_{n}\right)=\mathcal{S}_{n}$.

Since any relative kernel of a finite monoid $M$ is a submonoid of $M$, as a consequence of Remark 1.6 and previous theorem we have:

COROLlary 2.6. Let $\pi$ be an odd supernatural number and let $H_{\pi}$ be the corresponding pseudovariety of Abelian groups. Let $M$ be a finite monoid all of whose maximal subgroups are symmetric or dihedral groups. If $M$ is generated by its groupelements, then $\mathrm{K}_{\mathrm{H}_{\pi}}(M)=M$.

\section{ON PRESENTATIONS OF SOME TRANSFORMATION MONOIDS}

In this section we give some background on the inverse monoids whose relative Abelian kernels will be described.

To avoid ambiguities, from now on we take $n \geqslant 4$. Notice that, for $n \leqslant 3$, the relative Abelian kernels of the finitely many semigroups under consideration can be easily computed using the already referred implementation in GAP [27] of the algorithm presented in [4].

The reader can find more details and the proofs of the facts presented in this section in $[13,14,15,16,17]$.

3.1. The INVERSE SYMMETRIC MONOID $\mathcal{I}_{n}$. We begin by recalling some well known facts on the symmetric inverse monoid $\mathcal{I}_{n}$ on a base set with $n$ elements, that is, the inverse monoid (under composition) of all injective partial transformations on a set with $n$ elements.

Notice that the symmetric group $\mathcal{S}_{n}$ is the group of units of the monoid $\mathcal{I}_{n}$ and that two elements of $\mathcal{I}_{n}$ are $\mathfrak{R}$-related or $\mathfrak{L}$-related if they have the same domain or 
the same image, respectively. Moreover, given $s, t \in \mathcal{I}_{n}$, we have $s \leqslant_{\mathfrak{J}} t$ if and only if $|\operatorname{Im}(s)| \leqslant|\operatorname{Im}(t)|$. Hence, for $k \in\{0,1, \ldots, n\}$, being $J_{k}=\left\{s \in \mathcal{I}_{n}|| \operatorname{Im}(s) \mid=k\right\}$, we have

$$
\mathcal{I}_{n} / \mathfrak{J}=\left\{J_{0}<_{\mathfrak{J}} J_{1}<_{\mathfrak{J}} \cdots<_{\mathfrak{J}} J_{n}\right\}
$$

Since $\left|J_{k}\right|=\left(\begin{array}{l}n \\ k\end{array}\right)^{2} k !$, for $k \in\{0,1, \ldots, n\}$, it follows that $\mathcal{I}_{n}$ has $\sum_{k=0}^{n}\left(\begin{array}{l}n \\ k\end{array}\right)^{2} k$ ! elements. Observe that $J_{n}=\mathcal{S}_{n}$. Moreover, the maximal subgroups of $\mathcal{I}_{n}$ contained in $J_{k}$ are isomorphic to $\mathcal{S}_{k}$, for $1 \leqslant k \leqslant n$. We obtain a generating set of $\mathcal{I}_{n}$, with three elements, by joining to the permutations $a=(12)$ and $g=(12 \cdots n)$, which generate $\mathcal{S}_{n}$, any injective partial transformation of rank $n-1$. For instance, if

$$
c=\left(\begin{array}{cccc}
2 & 3 & \cdots & n \\
2 & 3 & \cdots & n
\end{array}\right)
$$

then the set $\{a, g, c\}$ generates the monoid $\mathcal{I}_{n}$. In particular, we have the following:

COROLlaRY 3.1. The inverse symmetric monoid $I_{n}$ is generated by its groupelements.

Combining the monoid presentation for $\mathcal{S}_{n}$ given in Example 2.1 with the Popova presentation of $\mathcal{I}_{n}[\mathbf{2 2}]$, one can deduce [16] the following presentation of $\mathcal{I}_{n}$, in terms of the generators $a, g$ and $c$ :

$$
\begin{aligned}
\langle a, g, c| a^{2}=g^{n}=(g a)^{n-1}=\left(a g^{n-1} a g\right)^{3} & =\left(a g^{n-j} a g^{j}\right)^{2}=1(2 \leqslant j \leqslant n-2), \\
g^{n-1} a g c g^{n-1} a g & \left.=g a c a g^{n-1}=c=c^{2},(c a)^{2}=c a c=(a c)^{2}\right\rangle .
\end{aligned}
$$

3.2. SOME INVERSE SUBMONOIDS OF $\mathcal{I}_{n}$. A partial transformation $\sigma$ of a chain $X_{n}$ with $n$ elements, say $X_{n}=\{1<2<\cdots<n\}$, is called order-preserving [order-reversing] if, $x \leqslant y$ implies $x \sigma \leqslant y \sigma[x \sigma \geqslant y \sigma]$, for all $x, y \in \operatorname{Dom}(\sigma)$. We denote by $\mathcal{P} O \mathcal{I}_{n}$ the inverse submonoid of $\mathcal{I}_{n}$ of all order-preserving transformations and by $\mathcal{P O D I} \mathcal{I}_{n}$ the inverse submonoid of $\mathcal{I}_{n}$ whose elements are all order-preserving or order-reversing transformations.

Let $c=\left(c_{1}, c_{2}, \ldots, c_{t}\right)$ be a sequence of $t(t \geqslant 0)$ elements from the chain $X_{n}$. We say that $c$ is cyclic [anti-cyclic] if there exists no more than one index $i \in\{1, \ldots, t\}$ such that $c_{i}>c_{i+1}\left[c_{i}<c_{i+1}\right]$, where $c_{i+1}=c_{1}$. Then, given a partial transformation $\sigma$ on the chain $X_{n}$ such that $\operatorname{Dom}(\sigma)=\left\{a_{1}, \ldots, a_{t}\right\}$, with $t \geqslant 0$ and $a_{1}<\cdots<a_{t}$, we say that $\sigma$ is orientation-preserving [orientation-reversing] if the sequence of its images $\left(a_{1} \sigma, \ldots, a_{t} \sigma\right)$ is cyclic [anti-cyclic]. We denote by $\mathcal{P} \mathcal{O P} \mathcal{I}_{n}$ the inverse submonoid of $\mathcal{I}_{n}$ of all orientation-preserving transformations and by $\mathcal{P O R \mathcal { I } _ { n }}$ the inverse submonoid of $\mathcal{I}_{n}$ of all orientation-preserving transformations together with all orientation-reversing transformations.

Notice that, $\mathcal{P O I _ { n }} \subset \mathcal{P O D I _ { n }} \subset \mathcal{P O R I _ { n }}$ and $\mathcal{P O I _ { n }} \subset \mathcal{P O P I _ { n }} \subset \mathcal{P O R I _ { n }}$, by definition. 


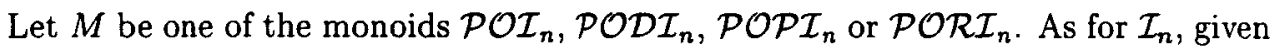
two elements $s, t \in M$, we have $s \leqslant \mathfrak{J} t$ if and only if $|\operatorname{Im}(s)| \leqslant|\operatorname{Im}(t)|$, hence

$$
M / \mathfrak{J}=\left\{J_{0}<_{\mathfrak{J}} J_{1}<_{\mathfrak{J}} \cdots<_{\mathfrak{J}} J_{n}\right\},
$$

where $J_{k}=\{s \in M:|\operatorname{Im}(s)|=k\}$, for $0 \leqslant k \leqslant n$.

Concerning maximal subgroups, the monoid $\mathcal{P O I}_{n}$ is aperiodic, while each $\mathfrak{H}$-class of an element $s \in \mathcal{P O D} \mathcal{I}_{n}$ has exactly two elements (an order-preserving one and another being order-reversing), unless the rank of $s$ is one or zero, in which case its $\mathfrak{H}$-class is trivial. On the other hand, for $1 \leqslant k \leqslant n$, the $\mathfrak{H}$-class of an element $s \in \mathcal{P O P} \mathcal{P} \mathcal{I}_{n}$ of rank $k$ has precisely $k$ elements, being a cyclic group of order $k$ if $s$ is a group-element. Finally, given $s \in \mathcal{P O R} \mathcal{I}_{n}$, if $|\operatorname{Im}(s)|=k \geqslant 3$, then the $\mathfrak{H}$-class of $s$ has $2 k$ elements and, if $s$ is a group-element, it is isomorphic to the dihedral group $D_{2 k}$ and if $|\operatorname{Im}(s)|=2$ then $\mathfrak{H}$-class of $s$ has precisely two elements, otherwise it has just one element.

Next, let us consider the elements $x_{0}, x_{1}, \ldots, x_{n-1}$ of $\mathcal{P O \mathcal { I } _ { n }}$ defined by:

$$
\begin{aligned}
& \text { 1. } x_{0}=\left(\begin{array}{cccc}
2 & \cdots & n-1 & n \\
1 & \cdots & n-2 & n-1
\end{array}\right)
\end{aligned}
$$

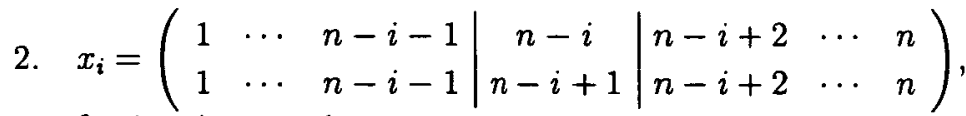

$$
\begin{aligned}
& \text { for } 1 \leqslant i \leqslant n-1 \text {. }
\end{aligned}
$$

Consider also the permutation (that reverts the order)

$$
h=\left(\begin{array}{ccccc}
1 & 2 & \cdots & n-1 & n \\
n & n-1 & \cdots & 2 & 1
\end{array}\right)
$$

of $\mathcal{P O D I} \mathcal{I}_{n}$ and the $n$-cycle

$$
g=\left(\begin{array}{ccccc}
1 & 2 & \cdots & n-1 & n \\
2 & 3 & \cdots & n & 1
\end{array}\right),
$$

which is an element of $\mathcal{P O P} \mathcal{I}_{n}$. Hence, $A=\left\{x_{0}, x_{1}, \ldots, x_{n-1}\right\}, B=A \cup\{h\}, C=A \cup\{g\}$

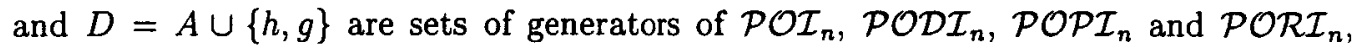
respectively.

Furthermore, consider the following set of monoid relations:

$$
\begin{array}{ll}
R_{1} & x_{i} x_{0}=x_{0} x_{i+1}, 1 \leqslant i \leqslant n-2 ; \\
R_{2} & x_{j} x_{i}=x_{i} x_{j}, 2 \leqslant i+1<j \leqslant n-1 ; \\
R_{3} & x_{0}^{2} x_{1}=x_{0}^{2}=x_{n-1} x_{0}^{2} ; \\
R_{4} & x_{i+1} x_{i} x_{i+1}=x_{i+1} x_{i}=x_{i} x_{i+1} x_{i}, 1 \leqslant i \leqslant n-2 ; \\
R_{5} & x_{i} x_{i+1} \cdots x_{n-1} x_{0} x_{1} \cdots x_{i-1} x_{i}=x_{i}, 0 \leqslant i \leqslant n-1 ; \\
R_{6} & x_{i+1} \cdots x_{n-1} x_{0} x_{1} \cdots x_{i-1} x_{i}^{2}=x_{i}^{2}, 1 \leqslant i \leqslant n-1 ;
\end{array}
$$




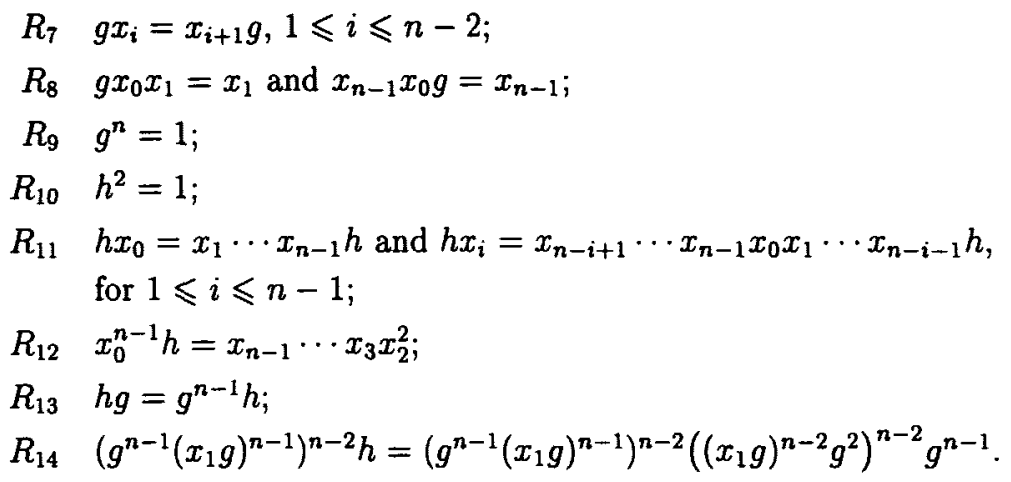

Observe that, we are adopting the following convention: given $i, j \in\{0, \ldots, n-1\}$, if $i \leqslant j$ the expression $x_{i} \cdots x_{j}$ represents the word of length $j-i+1$ such that the letter in the position $p \in\{1, \ldots, j-i+1\}$ is $x_{i+p-1}$ (that is, the indices of the letters are ordered in the usual way and are consecutive), and if $j<i$ the expression $x_{i} \cdots x_{j}$ represents the empty word. For example, the expression $x_{3} \cdots x_{2}$ denotes the empty word.

Hence, $\left\langle A \mid R_{1}-R_{6}\right\rangle,\left\langle B \mid R_{1}-R_{6}, R_{10}-R_{12}\right\rangle,\left\langle C \mid R_{1}-R_{9}\right\rangle$ and $\left\langle D \mid R_{1}-R_{14}\right\rangle$

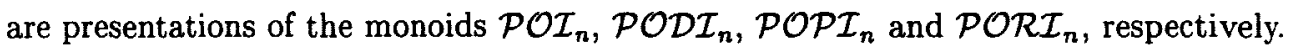

Next, we recall a set of canonical words associated to each of these presentations.

Let $k \in\{1, \ldots, n-1\}, \ell=n-k(1 \leqslant \ell \leqslant n-1)$ and $w_{j}=x_{\ell-j+1} \cdots x_{\ell-j+k}$, for $1 \leqslant j \leqslant \ell$. Notice that $\left|w_{j}\right|=k$, for $1 \leqslant j \leqslant \ell$. Let $A_{k}\left[C_{k}\right]$ be the set of all words

$$
\left(\prod_{j=1}^{\ell} u_{j}\right) x_{0}^{\ell}\left(\prod_{j=1}^{\ell} v_{j}\right)\left[g^{i}\left(\prod_{j=1}^{\ell} u_{j}\right) x_{0}^{\ell}\left(\prod_{j=1}^{\ell} v_{j}\right)\right],
$$

where $[0 \leqslant i \leqslant n-1], u_{j}$ is a suffix of $w_{j}$ and $v_{j}$ is a prefix of $w_{j}$, for $1 \leqslant j \leqslant \ell$, $0 \leqslant\left|u_{1}\right| \leqslant \cdots \leqslant\left|u_{\ell}\right| \leqslant k\left[1 \leqslant\left|u_{1}\right| \leqslant \cdots \leqslant\left|u_{\ell}\right| \leqslant k\right]$ and $k \geqslant\left|v_{1}\right| \geqslant \cdots \geqslant\left|v_{\ell}\right| \geqslant 0$. Also, define $A_{0}=C_{0}=\left\{x_{0}^{n}\right\}, A_{n}=\{1\}$ and $C_{n}=\left\{g^{i} \mid 0 \leqslant i \leqslant n-1\right\}$. Then $\bar{A}=A_{0} \cup A_{1} \cup \cdots \cup A_{n}$ and $\bar{C}=C_{0} \cup C_{1} \cup \cdots \cup C_{n}$ are sets of canonical words for $\mathcal{P O I}_{n}$ and $\mathcal{P O P} \mathcal{I}_{n}$, respectively.

Now, let $B_{k}=A_{k} \cup\left\{w h \mid w \in A_{k}\right\}$, for $2 \leqslant k \leqslant n$, and $B_{k}=A_{k}$, for $k=0$, 1. Also, define $D_{k}=C_{k} \cup\left\{w h \mid w \in C_{k}\right\}$, for $3 \leqslant k \leqslant n$, and $D_{k}=C_{k}$, for $0 \leqslant k \leqslant 2$. Then $\bar{B}=B_{0} \cup B_{1} \cup \cdots \cup B_{n}$ and $\bar{D}=D_{0} \cup D_{1} \cup \cdots \cup D_{n}$ are sets of canonical words for $\mathcal{P O D I _ { n }}$ and $\mathcal{P O R I _ { n }}$, respectively.

Notice that, for $0 \leqslant k \leqslant n$, the sets of words $A_{k}, B_{k}, C_{k}$ and $D_{k}$ represent the transformations of rank $k$ of $\mathcal{P O} \mathcal{I}_{n}, \mathcal{P O D} \mathcal{I}_{n}, \mathcal{P O P \mathcal { I } _ { n }}$ and $\mathcal{P O R \mathcal { I } _ { n }}$, respectively.

Of particular interest for us, are the words corresponding to elements of rank $n-1$ :

$$
\begin{aligned}
& A_{n-1}=\left\{x_{i} x_{i+1} \cdots x_{n-1} x_{0} x_{1} \cdots x_{j-1} \mid 1 \leqslant i \leqslant n, 1 \leqslant j \leqslant n\right\}, \\
& B_{n-1}=\left\{x_{i} x_{i+1} \cdots x_{n-1} x_{0} x_{1} \cdots x_{j-1} h^{t} \mid 1 \leqslant i \leqslant n, 1 \leqslant j \leqslant n, t=0,1\right\}, \\
& C_{n-1}=\left\{g^{r} x_{i} x_{i+1} \cdots x_{n-1} x_{0} x_{1} \cdots x_{j-1} \mid 1 \leqslant i \leqslant n-1,1 \leqslant j \leqslant n, 0 \leqslant r \leqslant n-1\right\},
\end{aligned}
$$




$$
\begin{aligned}
D_{n-1}= & \left\{g^{r} x_{i} x_{i+1} \cdots x_{n-1} x_{0} x_{1} \cdots x_{j-1} h^{t} \mid 1 \leqslant i \leqslant n-1,1 \leqslant j \leqslant n, 0 \leqslant r\right. \\
& \leqslant n-1, t=0,1\} .
\end{aligned}
$$

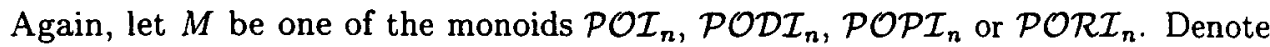
by $X$ the set of generators of $M$ and by $W$ the set of canonical words of $M$ considered above. Let $\varphi: X^{*} \longrightarrow M$ be the onto homomorphism extending the map $X \longrightarrow M$, $x \mapsto x$.

Given an element $s \in M$, we denote by $\mathrm{w}_{s}$ the (unique) element of $\varphi^{-1}(s) \cap W$, called the canonical word associated to $s$.

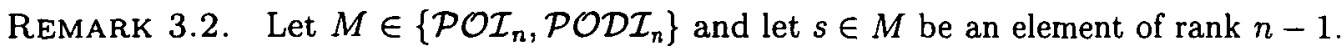
Then $s$ is an idempotent if and only if there exists $i \in\{0, \ldots, n-1\}$ such that

$$
\mathrm{w}_{s}=x_{i} \cdots x_{n-1} x_{0} x_{1} \cdots x_{i-1} .
$$

Notice that, if $s$ is not an idempotent, then $\left|w_{s}\right|_{x_{0}}=1$ and there exists $i \in\{1, \ldots, n$ - 1\} such that either $\left|w_{s}\right|_{x_{i}}=0$ or $\left|w_{s}\right|_{x_{i}}=2$.

On the other hand, if $M \in\left\{\mathcal{P O P} \mathcal{I}_{n}, \mathcal{P O R \mathcal { I } _ { n } \}}\right.$ and $s \in M$ is an element of rank $n-1$, then $s$ is an idempotent if and only if $\mathrm{w}_{s}=g^{n-1} x_{1} \cdots x_{n-1}$ (which corresponds to the same element of $M$ that the word $\left.x_{0} x_{1} \cdots x_{n-1}\right)$ or there exists $i \in\{1, \ldots, n-1\}$ such that

$$
\mathrm{w}_{s}=x_{i} \cdots x_{n-1} x_{0} x_{1} \cdots x_{i-1} .
$$

It is known (see [17]) that $\mathcal{P O R \mathcal { I } _ { n }}$ is generated by the transformations $g, h$ and $x_{1}$. Therefore $\left\{g, h, x_{1} g\right\}$ is also a set of generators of $\mathcal{P O R} \mathcal{I}_{n}$. As

$$
x_{1} g=\left(\begin{array}{ccccc}
1 & 2 & \cdots & n-2 & n-1 \\
2 & 3 & \cdots & n-1 & 1
\end{array}\right)
$$

is a partial permutation, we obtain the following:

COROLlary 3.3. The monoid $\mathcal{P O R} \mathcal{I}_{n}$ is generated by its group-elements.

\section{MAIN RESUltS}

This section is devoted to our main results. We give descriptions of the kernels relative to decidable pseudovarieties of Abelian groups of the monoids $\mathcal{P O I _ { n }}, \mathcal{P O D \mathcal { I } _ { n }}$, $\mathcal{P O P \mathcal { I } _ { n }}, \mathcal{P O R \mathcal { I } _ { n }}$ and $\mathcal{I}_{n}$ for which we already recalled simple presentations. As the kernel of a finite monoid relative to the trivial pseudovariety is the monoid itself, we just need to consider pseudovarieties of Abelian groups corresponding to infinite recursive supernatural numbers or to natural numbers greater than 1 .

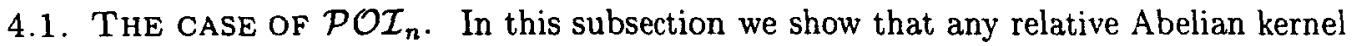
of $\mathcal{P O I _ { n }}$ equals the Abelian kernel of $\mathcal{P O I _ { n }}$. 
THEOREM 4.1. ([7]) The Abelian kernel of $\mathcal{P O} \mathcal{I}_{n}$ consists of all idempotents and all elements of rank less than $n-1$.

The Abelian kernel of $\mathcal{P O I _ { n }}$ contains all elements of rank less than $n-1$, so it must be the case of any relative Abelian kernel of $\mathcal{P O \mathcal { I } _ { n }}$.

Now, let $x \in \mathcal{P O} \mathcal{I}_{n}$ be an element of rank $n-1$. In [7, p. 445] it was proved that (considering the letters ordered as follows: $x_{0}<x_{1}<\cdots<x_{n-1}$ )

$$
\mathrm{Cl}_{\mathrm{Ab}}\left(\gamma\left(\varphi^{-1}(x)\right)\right) \subseteq \gamma\left(\mathrm{w}_{x}\right)+(1, \ldots, 1) \mathbb{Z}
$$

thus, using Proposition 1.12, for any $k>1$, we have

$$
\gamma_{k}\left(\varphi^{-1}(x)\right) \subseteq \gamma_{k}\left(w_{x}\right)+(1, \ldots, 1) \mathbb{Z} / k \mathbb{Z}
$$

By Remark 3.2, if $x$ is not idempotent, then $\gamma\left(w_{x}\right)$ has the first component 1 and the other components are 0 or 2, thus the same happens with $\gamma_{k}\left(w_{x}\right)$ (except that if $k=2$ none of the components is 2) and so $0 \notin \gamma_{k}\left(\varphi^{-1}(x)\right)$. It follows that the $\mathrm{K}_{\mathrm{H}_{k}}\left(\mathcal{P O I}_{n}\right)$ $=\mathrm{K}_{\mathrm{Ab}}\left(\mathcal{P} \mathcal{O} \mathcal{I}_{n}\right)$. Moreover, this equality holds even when the supernatural number under consideration is not finite:

THEOREM 4.2. Let $\pi \neq 1$ be a supernatural number. Then $\mathrm{K}_{\mathrm{H}_{\pi}}\left(\mathcal{P O} \mathcal{I}_{n}\right)$ $=\mathrm{K}_{\mathrm{Ab}}\left(\mathcal{P O} \mathcal{I}_{n}\right)$.

Proof: Suppose that $k>1$ is a finite divisor of $\pi$. As $\mathrm{H}_{k} \subseteq \mathrm{H}_{\pi}$, we have that

$$
\mathrm{K}_{\mathrm{Ab}}\left(\mathcal{P O} \mathcal{I}_{n}\right) \subseteq \mathrm{K}_{\mathrm{H}_{\pi}}\left(\mathcal{P O} \mathcal{I}_{n}\right) \subseteq \mathrm{K}_{\mathrm{H}_{\mathrm{k}}}\left(\mathcal{P O} \mathcal{I}_{n}\right)=\mathrm{K}_{\mathrm{Ab}}\left(\mathcal{P O} \mathcal{I}_{n}\right) \text {. }
$$

Thus the inclusions must in fact be equalities, concluding the proof.

4.2. THE CASE OF $\mathcal{P O D I _ { n }}$. In this subsection we compute the relative Abelian kernels of the monoid $\mathcal{P O D} \mathcal{I}_{n}$.

First we recall:

THEOREM 4.3. [9] If $n$ is an even integer, the Abelian kernel of $\mathcal{P O D} \mathcal{I}_{n}$ consists precisely of the elements of the Abelian kernel of $\mathcal{P O I _ { n }}$. If $n$ is an odd integer, then the Abelian kernel of $\mathcal{P O D I _ { n }}$ consists of the Abelian kernel of $\mathcal{P O} \mathcal{I}_{n}$ united with the set of elements corresponding to words of the form $x_{i} \cdots x_{n-1} x_{0} x_{1} \cdots x_{n-i}$, for $1 \leqslant i \leqslant n$.

Considering the presentation of $\mathcal{P O D I _ { n }}$ recalled in Section 3 , as an immediate consequence of Theorem 1.15, we have the following corollary.

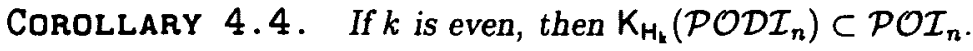

Let $\pi$ be a supernatural number.

Let $J_{i}$ denote the $\mathfrak{J}$-class of $\mathcal{P O D} \mathcal{I}_{n}$ of the elements of rank $i$, for $0 \leqslant i \leqslant n$.

As the group of units $J_{n}$ of $\mathcal{P O D} I_{n}$ is the cyclic group generated by the permutation of order two

$$
h=\left(\begin{array}{ccccc}
1 & 2 & \cdots & n-1 & n \\
n & n-1 & \cdots & 2 & 1
\end{array}\right),
$$


by Theorem 2.5 and Lemma 1.7 , we have

$$
\mathrm{K}_{\mathbf{H}_{\pi}}\left(\mathcal{P O O D} \mathcal{I}_{n}\right) \cap J_{n}=\mathbf{K}_{\mathbf{H}_{\pi}}\left(J_{n}\right)=\left\langle h^{\operatorname{gcd}(2, \pi)}\right\rangle= \begin{cases}\{1\}, & \text { if } 2 \text { divides } \pi \\ \{1, h\}, & \text { otherwise. }\end{cases}
$$

Next, we concentrate on the elements of rank less than $n-1$. First, we notice that, by Theorems 4.3 and $4.1, \mathcal{P O} \mathcal{I}_{n} \cap\left(\bigcup_{i=0}^{n-2} J_{i}\right)$ is contained in the Abelian kernel of $\mathcal{P O D \mathcal { I } _ { n }}$ and so it is contained in $\mathrm{K}_{\mathrm{H}_{\pi}}\left(\mathcal{P O D} \mathcal{I}_{n}\right)$.

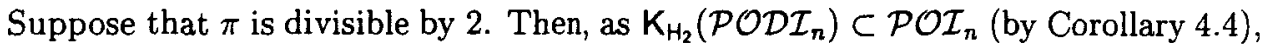
we have

$$
\begin{aligned}
\mathcal{P O} \mathcal{I}_{n} \cap\left(\bigcup_{i=0}^{n-2} J_{i}\right) & \subseteq \mathrm{K}_{\mathrm{H}_{\pi}}\left(\mathcal{P O D} \mathcal{I}_{n}\right) \cap\left(\bigcup_{i=0}^{n-2} J_{i}\right) \subseteq \mathrm{K}_{\mathrm{H}_{2}}\left(\mathcal{P O D} \mathcal{I}_{n}\right) \cap\left(\bigcup_{i=0}^{n-2} J_{i}\right) \\
& \subseteq \mathcal{P O} \mathcal{I}_{n} \cap\left(\bigcup_{i=0}^{n-2} J_{i}\right),
\end{aligned}
$$

hence $\mathrm{K}_{\mathrm{H}_{\pi}}\left(\mathcal{P} \mathcal{O D} \mathcal{I}_{n}\right) \cap\left(\bigcup_{i=0}^{n-2} J_{i}\right)=\mathcal{P O} \mathcal{I}_{n} \cap\left(\bigcup_{i=0}^{n-2} J_{i}\right)$.

On the other hand, admit that 2 does not divide $\pi$. Then

$$
\left(\mathcal{P O} \mathcal{I}_{n} \cap\left(\bigcup_{i=0}^{n-2} J_{i}\right)\right) \cup\{h\} \subset K_{H_{\pi}}\left(\mathcal{P O D} \mathcal{I}_{n}\right)
$$

and, since any element of $\mathcal{P O D} \mathcal{I}_{n}$ can be factorised as a product of a certain element of $\mathcal{P O I _ { n }}$ (with the same rank) by $h$, it follows that $\bigcup_{i=0}^{n-2} J_{i} \subset \mathrm{K}_{\mathrm{H}_{\pi}}\left(\mathcal{P O D} \mathcal{I}_{n}\right)$.

Summarising, we have

$$
\mathrm{K}_{H_{\pi}}\left(\mathcal{P} \mathcal{O D} \mathcal{I}_{n}\right) \cap\left(\bigcup_{i=0}^{n-2} J_{i}\right)= \begin{cases}\mathcal{P O} \mathcal{I}_{n} \cap\left(\bigcup_{i=0}^{n-2} J_{i}\right), & \text { if } 2 \text { divides } \pi \\ \bigcup_{i=0}^{n-2} J_{i}, & \text { otherwise. }\end{cases}
$$

Now, we just have to determine which elements of $J_{n-1}$ belong to $\mathrm{K}_{\mathrm{H}_{\pi}}\left(\mathcal{P O D} \mathcal{I}_{n}\right)$.

We recall that

$$
\left\{w_{i, j, t}=x_{i} x_{i+1} \cdots x_{n-1} x_{0} x_{1} \cdots x_{j-1} h^{t} \mid 1 \leqslant i \leqslant n, 1 \leqslant j \leqslant n, 0 \leqslant t \leqslant 1\right\}
$$

is a set of canonical words for $J_{n-1}$.

It is clear that

$$
\gamma\left(\mathbf{w}_{i, j, t}\right)= \begin{cases}\left(1, \ldots, 1,0_{(j+1)}, \ldots, 0_{(i)}, 1, \ldots, 1, t\right), & 1 \leqslant j \leqslant i \leqslant n \\ \left(1, \ldots, 1,2_{(i+1)}, \ldots, 2_{(j)}, 1, \ldots, 1, t\right), & 1 \leqslant i<j \leqslant n\end{cases}
$$


for $t=0,1$ (considering the letters ordered as follows: $x_{0}<x_{1}<\cdots<x_{n-1}<h$ ).

Let $x \in \mathcal{P O D I _ { n }}$ be an element of rank $n-1$. The second and third authors showed in [9] that $x \in \mathrm{K}_{\mathrm{Ab}}\left(\mathcal{P O D} \mathcal{I}_{n}\right)$ if and only if

$$
\begin{aligned}
\gamma\left(w_{x}\right) \in & (1,1, \ldots, 1,0) \mathbb{Z}+(0,0, \ldots, 0,2) \mathbb{Z}+(2,0, \ldots, 0) \mathbb{Z} \\
& +(0,1,0, \ldots, 0,1,0) \mathbb{Z}+\cdots+\left(0, \ldots, 0,1_{((n-1) / 2+1)}, 1_{((n+1) / 2+1)}, 0, \ldots, 0\right) \mathbb{Z},
\end{aligned}
$$

if $n$ is odd, and

$$
\begin{aligned}
\gamma\left(\mathbf{w}_{x}\right) \in & (1,1, \ldots, 1,0) \mathbb{Z}+(0,0, \ldots, 0,2) \mathbb{Z}+(2,0, \ldots, 0) \mathbb{Z} \\
& +(0,1,0, \ldots, 0,1,0) \mathbb{Z}+\cdots+\left(0, \ldots, 0,1_{n / 2}, 0,1_{(n / 2+2)}, 0, \ldots, 0\right) \mathbb{Z} \\
& +\left(0, \ldots, 0,2_{(n / 2+1)}, 0, \ldots, 0\right) \mathbb{Z},
\end{aligned}
$$

if $n$ is even. Notice that these expressions were deduced from the presentation of $\mathcal{P O D} \mathcal{I}_{n}$ [17] recalled in Section 3.

Hence, $x \in \mathrm{K}_{\mathrm{H}_{2}}\left(\mathcal{P O D \mathcal { I } _ { n } )}\right.$ if and only if $\gamma_{2}\left(\mathrm{w}_{x}\right)$ belongs to

$$
\begin{gathered}
(1,1, \ldots, 1,0) \mathbb{Z} / 2 \mathbb{Z}+ \\
(0,1,0, \ldots, 0,1,0) \mathbb{Z} / 2 \mathbb{Z}+\cdots+\left(0, \ldots, 0,1_{((n-1) / 2+1)}, 1_{((n+1) / 2+1)}, 0, \ldots, 0\right) \mathbb{Z} / 2 \mathbb{Z},
\end{gathered}
$$

if $n$ is odd, and to

$$
\begin{gathered}
(1,1, \ldots, 1,0) \mathbb{Z} / 2 \mathbb{Z}+ \\
(0,1,0, \ldots, 0,1,0) \mathbb{Z} / 2 \mathbb{Z}+\cdots+\left(0, \ldots, 0,1_{n / 2}, 0,1_{(n / 2+2)}, 0, \ldots, 0\right) \mathbb{Z} / 2 \mathbb{Z}
\end{gathered}
$$

if $n$ is even.

Now, suppose that $\mathbf{w}_{x}=\mathbf{w}_{i, j, t}$, for some $1 \leqslant i \leqslant n, 1 \leqslant j \leqslant n$ and $0 \leqslant t \leqslant 1$. If $n$ is even, then it is clear that $x \in \mathrm{K}_{\mathrm{H}_{2}}\left(\mathcal{P O D \mathcal { I } _ { n } )}\right.$ if and only if $i=j$ and $t=0$, that is, $x \in \mathrm{K}_{\mathrm{H}_{2}}\left(\mathcal{P O D} \mathcal{I}_{n}\right)$ if and only if $x$ is an idempotent of $J_{n-1}$. Thus $\mathrm{K}_{\mathrm{H}_{2}}\left(\mathcal{P O D} \mathcal{I}_{n}\right) \cap J_{n-1}$ $=\mathrm{K}_{\mathrm{Ab}}\left(\mathcal{P O D} \mathcal{I}_{n}\right) \cap J_{n-1}$. On the other hand, if $n$ is odd, then it is easy to show that

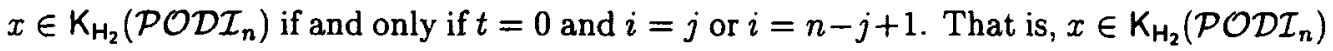
if and only if $x$ is an idempotent of $J_{n-1}$ or $x=x_{i} x_{i+1} \cdots x_{n-1} x_{0} x_{1} \cdots x_{n-i}$, for some $1 \leqslant i \leqslant n$. Thus we also have in this case, $\mathrm{K}_{\mathrm{H}_{2}}\left(\mathcal{P O D} \mathcal{I}_{n}\right) \cap J_{n-1}=\mathrm{K}_{\mathrm{Ab}}\left(\mathcal{P O D} \mathcal{I}_{n}\right) \cap J_{n-1}$.

Hence $\mathrm{K}_{\mathrm{H}_{2}}\left(\mathcal{P O D} \mathcal{I}_{n}\right)=\mathrm{K}_{\mathrm{Ab}}\left(\mathcal{P O D} \mathcal{I}_{n}\right)$, for all $n \in \mathbb{N}$, and so, using Proposition 1.9, we get the following result:

THEOREM 4.5. If $\pi$ is an even supernatural number, then $K_{\mathrm{H}_{\pi}}\left(\mathcal{P O D} \mathcal{I}_{n}\right)$ $=\mathrm{K}_{\mathrm{Ab}}\left(\mathcal{P} \mathcal{O D} \mathcal{I}_{n}\right)$.

Next, let $k$ be an odd natural number and consider again an element $x \in \mathcal{P O D} \mathcal{I}_{n}$ of rank $n-1$. As $2 \mathbb{Z} / k \mathbb{Z}=\mathbb{Z} / k \mathbb{Z}$, we have: 
(1) If $n$ is odd, then $x \in \mathrm{K}_{\mathrm{H}_{k}}\left(\mathcal{P O D} \mathcal{I}_{n}\right)$ if and only if

$$
\begin{aligned}
\gamma_{k}\left(\mathbf{w}_{x}\right) \in & (1,1, \ldots, 1,0) \mathbb{Z} / k \mathbb{Z}+(0,0, \ldots, 0,2) \mathbb{Z} / k \mathbb{Z}+(2,0, \ldots, 0) \mathbb{Z} / k \mathbb{Z} \\
& +(0,1,0, \ldots, 0,1,0) \mathbb{Z} / k \mathbb{Z}+\cdots+\left(0, \ldots, 0,1_{((n-1) / 2+1)}, 1_{((n+1) / 2+1)}, 0, \ldots, 0\right) \mathbb{Z} / k \mathbb{Z} \\
= & (1,1, \ldots, 1,0) \mathbb{Z} / k \mathbb{Z}+(0,0, \ldots, 0,1) \mathbb{Z} / k \mathbb{Z}+(1,0, \ldots, 0) \mathbb{Z} / k \mathbb{Z} \\
& +(0,1,0, \ldots, 0,1,0) \mathbb{Z} / k \mathbb{Z}+\cdots+\left(0, \ldots, 0,1_{((n-1) / 2+1)}, 1_{((n+1) / 2+1)}, 0, \ldots, 0\right) \mathbb{Z} / k \mathbb{Z} \\
= & (1,1, \ldots, 1,0) \mathbb{Z} / k \mathbb{Z}+(0,0, \ldots, 0,1) \mathbb{Z} / k \mathbb{Z} \\
& +(0,1,0, \ldots, 0,1,0) \mathbb{Z} / k \mathbb{Z}+\cdots+\left(0, \ldots, 0,1_{((n-1) / 2+1)}, 1_{((n+1) / 2+1)}, 0, \ldots, 0\right) \mathbb{Z} / k \mathbb{Z}
\end{aligned}
$$

(2) If $n$ is even, then $x \in \mathrm{K}_{\mathrm{H}_{\mathrm{k}}}\left(\mathcal{P O D} \mathcal{I}_{n}\right)$ if and only if

$$
\begin{aligned}
& \gamma_{k}\left(w_{x}\right) \in(1,1, \ldots, 1,0) \mathbb{Z} / k \mathbb{Z}+(0,0, \ldots, 0,2) \mathbb{Z} / k \mathbb{Z}+(2,0, \ldots, 0) \mathbb{Z} / k \mathbb{Z} \\
&+(0,1,0, \ldots, 0,1,0) \mathbb{Z} / k \mathbb{Z}+\cdots+\left(0, \ldots, 0,1_{n / 2}, 0,1_{(n / 2+2)}, 0, \ldots, 0\right) \mathbb{Z} / k \mathbb{Z} \\
&+\left(0, \ldots, 0,2_{(n / 2+1)}, 0, \ldots, 0\right) \mathbb{Z} / k \mathbb{Z} \\
&=(1,1, \ldots, 1,0) \mathbb{Z} / k \mathbb{Z}+(0,0, \ldots, 0,1) \mathbb{Z} / k \mathbb{Z}+(1,0, \ldots, 0) \mathbb{Z} / k \mathbb{Z} \\
& \quad+(0,1,0, \ldots, 0,1,0) \mathbb{Z} / k \mathbb{Z}+\cdots+\left(0, \ldots, 0,1_{(n / 2)}, 0,1_{(n / 2+2)}, 0, \ldots, 0\right) \mathbb{Z} / k \mathbb{Z} \\
& \quad+\left(0, \ldots, 0,1_{(n / 2+1)}, 0, \ldots, 0\right) \mathbb{Z} / k \mathbb{Z} \\
&=(1,1, \ldots, 1,0) \mathbb{Z} / k \mathbb{Z}+(0,0, \ldots, 0,1) \mathbb{Z} / k \mathbb{Z} \\
& \quad+(0,1,0, \ldots, 0,1,0) \mathbb{Z} / k \mathbb{Z}+\cdots+\left(0, \ldots, 0,1_{n / 2}, 0,1_{(n / 2+2)}, 0, \ldots, 0\right) \mathbb{Z} / k \mathbb{Z} \\
& \quad+\left(0, \ldots, 0,1_{(n / 2+1)}, 0, \ldots, 0\right) \mathbb{Z} / k \mathbb{Z} .
\end{aligned}
$$

Then, supposing that $w_{x}=w_{i, j, t}$, for some $1 \leqslant i \leqslant n, 1 \leqslant j \leqslant n$ and $0 \leqslant t \leqslant 1$, it is easy to show that, for both $n$ odd and even, we have $x \in \mathrm{K}_{\mathrm{H}_{\mathrm{k}}}\left(\mathcal{P O D} \mathcal{I}_{n}\right)$ if and only if $i=j$ or $i=n-j+1$. Hence, $x \in \mathrm{K}_{\mathrm{H}_{k}}\left(\mathcal{P O D} \mathcal{I}_{n}\right)$ if and only if $x=x_{i} x_{i+1} \cdots x_{n-1} x_{0} x_{1} \cdots x_{i-1} h^{t}$ or $x=x_{i} x_{i+1} \cdots x_{n-1} x_{0} x_{1} \cdots x_{n-i} h^{t}$, for some $1 \leqslant i \leqslant n$ and $0 \leqslant t \leqslant 1$.

Now, let $\pi$ be an odd supernatural. Then, $\pi$ is divisible by some odd natural number $k$ and we have $\mathrm{K}_{\mathrm{H}_{\pi}}\left(\mathcal{P O D} \mathcal{I}_{n}\right) \subseteq \mathrm{K}_{\mathrm{H}_{k}}\left(\mathcal{P O D} \mathcal{I}_{n}\right)$. In fact, the converse inclusion is also valid, as we shall show below.

First, notice that, by relations $R_{11}$, we have $x_{0} h=h x_{1} \cdots x_{n-1}$ and

$$
x_{n-1} h=h x_{i+1} \cdots x_{n-1} x_{0} x_{1} \cdots x_{i-1} .
$$

Hence

$$
x_{i} x_{i+1} \cdots x_{n-1} x_{0} x_{1} \cdots x_{n-i} h=x_{i} x_{i+1} \cdots x_{n-1} x_{0} x_{1} \cdots x_{n-i-1} h x_{i+1} \cdots x_{n-1} x_{0} x_{1} \cdots x_{i-1},
$$

for $1 \leqslant i \leqslant n-1$. It follows that, for $1 \leqslant i \leqslant n, x_{i} x_{i+1} \cdots x_{n-1} x_{0} x_{1} \cdots x_{i-1}$ and $x_{i} x_{i+1} \cdots x_{n-1} x_{0} x_{1} \cdots x_{n-i} h$ are $\mathfrak{H}$-related elements of $\mathcal{P O D} \mathcal{I}_{n}$, thus

$$
\left\{x_{i} x_{i+1} \cdots x_{n-1} x_{0} x_{1} \cdots x_{i-1}, x_{i} x_{i+1} \cdots x_{n-1} x_{0} x_{1} \cdots x_{n-i} h\right\}
$$

is a cyclic group of order two and so it is contained in $K_{H_{\pi}}\left(\mathcal{P O D} \mathcal{I}_{n}\right)$. Therefore, since $h \in \mathrm{K}_{\mathrm{H}_{\pi}}\left(\mathcal{P O D} \mathcal{I}_{n}\right)$, we have proved: 
THEOREM 4.6. If $\pi$ is an odd supernatural number, then $\mathrm{K}_{\mathrm{H}_{\pi}}\left(\mathcal{P O D} \mathcal{I}_{n}\right)$ consists of all permutations of $\mathcal{P O D I _ { n }}$, of all elements of rank $n-1$ of the forms $x_{i} x_{i+1} \cdots x_{n-1} x_{0} x_{1} \cdots x_{i-1} h^{t}$ and $x_{i} x_{i+1} \cdots x_{n-1} x_{0} x_{1} \cdots x_{n-i} h^{t}$, with $1 \leqslant i \leqslant n$ and $0 \leqslant t \leqslant 1$, and all transformations with rank less than $n-1$.

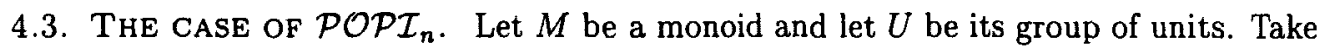
$x \in U$ and $s \in M$. Then, it is easy to show that $s \mathcal{L} x s$. Furthermore, as the Green relation $\mathfrak{R}$ is compatible with the multiplication on the left, the correspondence

$$
\begin{array}{ccc}
H_{s} & \longrightarrow & H_{x s} \\
t & \mapsto & x t
\end{array}
$$

is a bijection.

Next, let $g$ be the $n$-cycle permutation and $s$ any element of $\mathcal{I}_{n}$ with rank $n-1$. Then, $s, g s, g^{2} s, \ldots, g^{n-1} s$ are $n$ domain-distinct (and so each one lies in a different $\mathfrak{R}$ class of $\mathcal{I}_{n}$ ) $\mathfrak{L}$-related elements of $\mathcal{I}_{n}$ of rank $n-1$. Hence, $X, g X, g^{2} X, \ldots, g^{n-1} X$ are $n$ pairwise disjoint subsets of $L_{s}$ with $|X|$ elements each, for any subset $X$ of $H_{s}$.

Now, consider the monoid $\mathcal{P O P} \mathcal{I}_{n}$ and let $\pi$ be a supernatural number. Recall that:

THEOREM 4.7. ([7]) The Abelian kernel of $\mathcal{P O P} \mathcal{I}_{n}$ consists of all idempotents and all elements of rank less than $n-1$.

Let $J_{i}$ denote the $\mathfrak{J}$-class of $\mathcal{P} \mathcal{O P} \mathcal{I}_{n}$ of the elements of rank $i$, for $0 \leqslant i \leqslant n$.

As $J_{0}, J_{1}, \ldots, J_{n-2}$ are contained in the Abelian kernel of $\mathcal{P O P} \mathcal{I}_{n}$, we have also

$$
\bigcup_{i=0}^{n-2} J_{i} \subset K_{H_{n}}\left(\mathcal{P O P} \mathcal{I}_{n}\right)
$$

On the other hand, as the group of units $J_{n}$ of $\mathcal{P O P \mathcal { I } _ { n }}$ is the cyclic group generated by the permutation $g=(12 \cdots n)$, by Theorem 2.5 and by Lemma 1.7 , we have

$$
\mathrm{K}_{\mathrm{H}_{\pi}}\left(\mathcal{P} \mathcal{O P} \mathcal{I}_{n}\right) \cap J_{n}=\mathrm{K}_{\mathrm{H}_{\pi}}\left(J_{n}\right)=\left\langle g^{\mathrm{gcd}(n, \pi)}\right\rangle .
$$

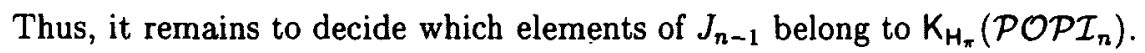

Let $H$ be a maximal subgroup of $\mathcal{P O P} \mathcal{I}_{n}$ contained in $J_{n-1}$. Then, $H$ is a cyclic group of order $n-1$ and so, by Theorem $2.5, \mathrm{~K}_{H_{\pi}}(H)$ has $(n-1) /(\operatorname{gcd}(n-1, \pi))$ elements. As

$$
\mathrm{K}_{\mathrm{H}_{*}}(H) \cup g^{\operatorname{gcd}(n, \pi)} \mathrm{K}_{\mathrm{H}_{\pi}}(H) \cup g^{2 \operatorname{gcd}(n, \pi)} \mathrm{K}_{\mathrm{H}_{\pi}}(H) \cup \cdots \cup g^{((n / \operatorname{gcd}(n, \pi))-1) \operatorname{gcd}(n, \pi)} \mathrm{K}_{\mathrm{H}_{\pi}}(H)
$$

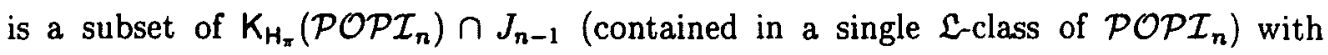
cardinality $n /(\operatorname{gcd}(n, \pi)) \cdot(n-1) /(\operatorname{gcd}(n-1, \pi))$ and $J_{n-1}$ contains $n$ distinct maximal subgroups of $\mathcal{P O P \mathcal { I } _ { n }}$ (and $\mathcal{P O P \mathcal { I } _ { n }}$ is an inverse monoid), we have at least

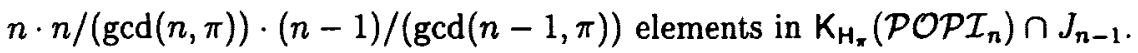

Now, let $k$ be any natural number. 
Recall that

$\left\{\mathrm{w}_{i, j, r}=g^{r} x_{i} x_{i+1} \cdots x_{n-1} x_{0} x_{1} \cdots x_{j-1} \mid 1 \leqslant i \leqslant n-1,1 \leqslant j \leqslant n, 0 \leqslant r \leqslant n-1\right\}$

is a set of canonical words for $J_{n-1}$. Clearly, for all $0 \leqslant r \leqslant n-1$, we have

$$
\gamma\left(w_{i, j, r}\right)=\left\{\begin{array}{ll}
\left(1, \ldots, 1,0_{(j+1)}, \ldots, 0_{(i)}, 1, \ldots, 1, r\right), & 1 \leqslant j \leqslant i \leqslant n-1 \\
\left(1, \ldots, 1,2_{(i+1)}, \ldots, 2_{(j)}, 1, \ldots, 1, r\right), & 1 \leqslant i<j \leqslant n
\end{array} .\right.
$$

(considering the letters ordered as follows: $x_{0}<x_{1}<\cdots<x_{n-1}<g$ ).

Let $x \in J_{n-1}$. In [7, Corollary 4.7], the second and third authors showed that

$$
\overline{\gamma\left(\psi^{-1}(x)\right)}=\gamma\left(\mathbf{w}_{x}\right)+(1,1, \ldots, 1,0) \mathbb{Z}+\sum_{i=1}^{n-2} f_{i} \mathbb{Z}+(1,0, \ldots, 0,1) \mathbb{Z}+(0,0, \ldots, 0, n) \mathbb{Z}
$$

where $f_{i}=\left(0_{(1)}, \ldots, 0,-1_{(i+1)}, 1_{(i+2)}, 0, \ldots, 0_{(n+1)}\right)$, for all $1 \leqslant i \leqslant n-2$. Notice that this formula was deduced from the presentation of $\mathcal{P O P} \mathcal{I}_{n}[14]$ recalled in the Section 3 . It follows that

$$
\begin{gathered}
\gamma_{k}\left(\psi^{-1}(x)\right)= \\
\gamma_{k}\left(\mathrm{w}_{x}\right)+(1,1, \ldots, 1,0) \mathbb{Z} / k \mathbb{Z}+\sum_{i=1}^{n-2} f_{i} \mathbb{Z} / k \mathbb{Z}+(1,0, \ldots, 0,1) \mathbb{Z} / k \mathbb{Z}+(0,0, \ldots, 0, n) \mathbb{Z} / k \mathbb{Z} .
\end{gathered}
$$

Next, assume that $x \in \mathrm{K}_{\mathrm{H}_{k}}\left(\mathcal{P} \mathcal{O P} \mathcal{I}_{n}\right)$. Hence $0 \in \gamma_{k}\left(\psi^{-1}(x)\right)$ and so the system of equations

$$
\gamma\left(\mathbf{w}_{x}\right)+(1,1, \ldots, 1,0) z_{0}+\sum_{i=1}^{n-2} f_{i} z_{i}+(1,0, \ldots, 0,1) z_{n-1}+(0,0, \ldots, 0, n) z_{n} \equiv 0 \quad \bmod k,
$$

with integer unknowns $z_{0}, z_{1}, \ldots, z_{n}$, has a solution. Let $\gamma\left(w_{x}\right)=\left(a_{0}, a_{1}, \ldots, a_{n-1}, a_{n}\right)$. Then, we have

$$
\left\{\begin{array}{l}
z_{0}+z_{n-1} \equiv-a_{0} \quad \bmod k \\
z_{0}-z_{1} \equiv-a_{1} \quad \bmod k \\
z_{0}+z_{i-1}-z_{i} \equiv-a_{i} \bmod k, 2 \leqslant i \leqslant n-2 \\
z_{0}+z_{n-2} \equiv-a_{n-1} \quad \bmod k \\
z_{n-1}+n z_{n} \equiv-a_{n} \bmod k .
\end{array}\right.
$$

From this system it is easy to deduce the following equation:

$$
(n-1)\left(z_{n-1}+a_{0}\right) \equiv\left(a_{1}+\cdots+a_{n-1}\right) \bmod k .
$$

Suppose that $w_{x}=w_{i, j, r}$, for some $1 \leqslant i \leqslant n-1,1 \leqslant j \leqslant n$ and $0 \leqslant r \leqslant n-1$. Then $a_{0}=1, a_{1}+\cdots+a_{n-1}=n-i+j-1$ and $a_{n}=r$. Hence, the system of equations

$$
\left\{\begin{array}{l}
(n-1)\left(z_{n-1}+1\right) \equiv(n-i+j-1) \bmod k \\
z_{n-1}+n z_{n} \equiv-r \bmod k
\end{array}\right.
$$


with integer unknowns $z_{n-1}$ and $z_{n}$, must have a solution. Now, from (1) we have

$$
\left\{\begin{array}{l}
(n-1) z_{n-1} \equiv(j-i) \quad \bmod k \\
(n-1) z_{n-1}+n(n-1) z_{n} \equiv-r(n-1) \quad \bmod k
\end{array}\right.
$$

and so the equation

$$
n(n-1) z_{n} \equiv-(r(n-1)+j-i) \bmod k
$$

has a solution, which implies that $\operatorname{gcd}(n(n-1), k)$ divides $r(n-1)+j-i$. It follows, by Proposition 1.22, that the set $\mathrm{K}_{\mathrm{H}_{k}}\left(\mathcal{P O P} \mathcal{I}_{n}\right) \cap J_{n-1}$ has at most

$$
\frac{n^{2}(n-1)}{\operatorname{gcd}(n(n-1), k}
$$

elements. Since $\operatorname{gcd}(n, n-1)=1$, then $\operatorname{gcd}(n(n-1), k)=\operatorname{gcd}(n, k) \operatorname{gcd}(n-1, k)$ and so we have precisely

$$
\left|\mathrm{K}_{\mathrm{H}_{\mathrm{k}}}\left(\mathcal{P} \mathcal{O} \mathcal{P} \mathcal{I}_{n}\right) \cap J_{n-1}\right|=\frac{n^{2}(n-1)}{\operatorname{gcd}(n(n-1), k)}
$$

Therefore we have:

THEOREM 4.8. For all $k \in \mathbb{N}$, the relative kernel $\mathrm{K}_{\mathrm{H}_{k}}\left(\mathcal{P O P} \mathcal{I}_{n}\right)$ consists of all permutations generated by $g^{\operatorname{gcd}(n, k)}$, of all elements of rank $n-1$ of the form $g^{r} x_{i} x_{i+1} \cdots x_{n-1} x_{0} x_{1} \cdots x_{j-1}$, with $1 \leqslant i \leqslant n-1,1 \leqslant j \leqslant n, 0 \leqslant r \leqslant n-1$ and $\operatorname{gcd}(n(n-1), k) \mid r(n-1)+j-i$, and all transformations with rank less than $n-1$.

Now, let $k=\operatorname{gcd}(n(n-1), \pi)$. Notice that, clearly, $\operatorname{gcd}(n, \pi)=\operatorname{gcd}(n, k), \operatorname{gcd}(n-$ $1, \pi)=\operatorname{gcd}(n-1, k)$ and $k=\operatorname{gcd}(n(n-1), k)=\operatorname{gcd}(n, k) \operatorname{gcd}(n-1, k)=\operatorname{gcd}(n, \pi) \operatorname{gcd}(n-$ $1, \pi)$, since $\operatorname{gcd}(n, n-1)=1$. Thus, in particular, we have

$$
\mathrm{K}_{\mathrm{H}_{n}}\left(\mathcal{P O P} \mathcal{I}_{n}\right) \cap J_{n}=\left\langle g^{\mathrm{gcd}(n, \pi)}\right\rangle=\left\langle g^{\mathrm{gcd}(n, k)}\right\rangle=\mathrm{K}_{\mathrm{H}_{k}}\left(\mathcal{P} \mathcal{O} \mathcal{P} \mathcal{I}_{n}\right) \cap J_{n}
$$

and (as $k$ divides $\pi$ )

$$
\mathrm{K}_{\mathrm{H}_{n}}\left(\mathcal{P O P} \mathcal{I}_{n}\right) \subseteq \mathrm{K}_{\mathrm{H}_{\mathrm{k}}}\left(\mathcal{P O P} \mathcal{I}_{n}\right)
$$

Moreover, since

$$
\begin{aligned}
\left|\mathrm{K}_{\mathrm{H}_{k}}\left(\mathcal{P O P} \mathcal{I}_{n}\right) \cap J_{n-1}\right| & =\frac{n^{2}(n-1)}{\operatorname{gcd}(n(n-1), k)}=\frac{n^{2}(n-1)}{\operatorname{gcd}(n, \pi) \operatorname{gcd}(n-1, \pi)} \\
& \leqslant\left|\mathrm{K}_{\mathrm{H}_{n}}\left(\mathcal{P O P} \mathcal{I}_{n}\right) \cap J_{n-1}\right|,
\end{aligned}
$$

we have proved the following theorem.

THEOREM 4.9. Let $\pi$ be a supernatural number. Then $\mathrm{K}_{\mathrm{H}_{z}}\left(\mathcal{P O P} \mathcal{I}_{n}\right)=\mathrm{K}_{\mathrm{H}_{k}}\left(\mathcal{P O P} \mathcal{I}_{n}\right)$, with $k=\operatorname{gcd}(n(n-1), \pi)$. 
4.4. The CASE of $\mathcal{P O R} \mathcal{I}_{n}$. First notice that Corollaries 3.3 and 2.6 combined with the fact that the maximal subgroups of $\mathcal{P O R} \mathcal{I}_{n}$ are dihedral groups allow us to conclude immediately:

PROPOSITION 4.10. If $\pi$ is an odd supernatural number, then $\mathrm{K}_{\mathrm{H}_{\pi}}\left(\mathcal{P O R} \mathcal{I}_{n}\right)$ $=\mathcal{P O R \mathcal { I } _ { n }}$.

Next we shall prove that $\mathrm{K}_{\mathrm{H}_{2}}\left(\mathcal{P O R} \mathcal{I}_{n}\right)=\mathrm{K}_{\mathrm{Ab}}\left(\mathcal{P O R I _ { n }}\right)$.

Recall that in [9] it was proved that the Abelian kernel of $\mathcal{P O R \mathcal { I } _ { n }}$ is contained in $\mathcal{P O P} \mathcal{I}_{n}$. Denote by $J_{i}$ the $\mathfrak{J}$-class of $\mathcal{P O R} \mathcal{I}_{n}$ of all elements of rank $i$, for $0 \leqslant i \leqslant n$. The following result gives a description of the elements of $\mathcal{P O R} \mathcal{I}_{n} \cap J_{n-1}$ that are in the Abelian kernel of $\mathcal{P O R} \mathcal{I}_{n}$.

Recall that $\left\{x_{0}, x_{1}, \ldots, x_{n-1}, h, g\right\}$ is a set of generators of $\mathcal{P O R} \mathcal{I}_{n}$.

THEOREM 4.11. [9] Let $X=\left\{x_{1}, \ldots, x_{n-1}\right\}$ and let $x$ be the element of $J_{n-1}$ $\cap \mathcal{P O P} \mathcal{I}_{n}$ corresponding to the word $w=g^{k}\left(x_{n-i} \cdots x_{n-1}\right) x_{0}\left(x_{1} \cdots x_{j}\right)$, with $0 \leqslant k$ $\leqslant n-1,1 \leqslant i \leqslant n-1$ and $0 \leqslant j \leqslant n-1$. Then $x \in \mathrm{K}_{\mathrm{Ab}}\left(\mathcal{P O R} \mathcal{I}_{n}\right)$ if and only if:

(i) $|w|_{X}$ is even, for $n$ odd;

(ii) $|w|$ is even, for $n$ even.

From Lemmas 1.7 and 2.4 we have that $\mathrm{K}_{\mathrm{Ab}}\left(\mathcal{P O R} \mathcal{I}_{n}\right) \cap J_{n}=\left\langle g^{2}\right\rangle$ and it was also observed in [9] that, for $k<n-1, \mathrm{~K}_{\mathrm{Ab}}\left(\mathcal{P O R \mathcal { I } _ { n }}\right) \cap J_{k}$ consists of the elements of $J_{k}$ that belong to $\mathcal{P O P} \mathcal{I}_{n}$.

Now, using the presentation of $\mathcal{P O R I _ { n }}$ recalled in Section 3, as an immediate consequence of Theorem 1.15, we have:

COROLLARY 4.12. If $k$ is even, then $\mathrm{K}_{\mathrm{H}_{k}}\left(\mathcal{P O R \mathcal { I } _ { n }}\right) \subset \mathcal{P O P \mathcal { I } _ { n }}$.

It follows from Proposition 1.14 that if $x \in \mathrm{K}_{\mathrm{H}_{2}}\left(\mathcal{P O R} \mathcal{I}_{n}\right)$ then there exists a word $w$ representing $x$ such that $|w|_{Y}$ is even, for all subset $Y$ of the set of generators, hence, $x \in \mathrm{K}_{\mathrm{Ab}}\left(\mathcal{P O R} \mathcal{I}_{n}\right)$, by Theorem 4.11. Therefore:

PROPOSITION 4.13. $\mathrm{K}_{\mathrm{H}_{2}}\left(\mathcal{P O R I _ { n }}\right)=\mathrm{K}_{\mathrm{Ab}}\left(\mathcal{P O R I _ { n }}\right)$.

By Propositions $4.10,4.13$ and 1.9, we get the main result of this subsection:

TheOREM 4.14. Let $\pi$ be a supernatural number and $\mathbf{H}_{\pi}$ the associated pseudovariety of Abelian groups. Then $\mathrm{K}_{\mathrm{H}_{\pi}}\left(\mathcal{P O R} \mathcal{I}_{n}\right)=\mathrm{K}_{\mathrm{Ab}}\left(\mathcal{P O R} \mathcal{I}_{n}\right)$ if $\pi$ is even and $K_{\mathrm{H}_{\pi}}\left(\mathcal{P O R \mathcal { I } _ { n }}\right)=\mathcal{P O R \mathcal { I } _ { n }}$ if $\pi$ is odd.

4.5. THE CASE OF $\mathcal{I}_{n}$. We start this subsection, as the previous one, by noticing that Corollaries 3.1 and 2.6 combined with the fact that the maximal subgroups of $\mathcal{I}_{n}$ are symmetric groups imply the following:

PRoposition 4.15. If $\pi$ is an odd supernatural number, then $K_{H_{\pi}}\left(\mathcal{I}_{n}\right)=\mathcal{I}_{n}$.

Now recall a description of the Abelian kernel of $\mathcal{I}_{n}$ given by the second and third authors [9]. 
TheOREM 4.16. The Abelian kernel of $\mathcal{I}_{n}$ consists of all even permutations of $\mathcal{S}_{n}$, of all the $\left|J_{n-1}\right| / 2$ elements (with rank $n-1$ ) of $\mathcal{A}_{n-1} H^{\prime} \mathcal{A}_{n-1}$, where $H$ is any maximal subgroup of $J_{n-1}$, and of all transformations with rank less than $n-1$.

Next we shall prove that $K_{H_{2}}\left(\mathcal{I}_{n}\right)=K_{A b}\left(\mathcal{I}_{n}\right)$. Notice that, by Theorem 2.5, the elements of rank $n$ belong to $\mathrm{K}_{\mathrm{H}_{2}}\left(\mathcal{I}_{n}\right)$ exactly when they belong to $\mathrm{K}_{\mathrm{Ab}}\left(\mathcal{I}_{n}\right)$. As $\mathrm{K}_{\mathrm{Ab}}\left(\mathcal{I}_{n}\right)$ contains all transformations with rank less than $n-1$ and is contained in $K_{\mathrm{H}_{2}}\left(\mathcal{I}_{n}\right)$, it remains to check that $\mathrm{K}_{\mathrm{H}_{2}}\left(\mathcal{I}_{n}\right) \cap J_{n-1}=\mathrm{K}_{\mathrm{Ab}}\left(\mathcal{I}_{n}\right) \cap J_{n-1}$. Since $\mathrm{K}_{\mathrm{Ab}}\left(\mathcal{I}_{n}\right) \subseteq \mathrm{K}_{\mathrm{H}_{2}}\left(\mathcal{I}_{n}\right)$, can be concluded by showing that both sets have the same number of elements, that is, by showing that $\mathrm{K}_{\mathrm{H}_{2}}\left(\mathcal{I}_{n}\right) \cap J_{n-1}$ has $\left|J_{n-1}\right| / 2$ elements. To achieve this we shall follow the strategy used in [9] to prove part of Theorem 4.16. With the same proof as [9, Lemma 6.1], we have the following lemma, which implies that all $\mathfrak{H}$-classes of $\mathcal{I}_{n} \cap J_{n-1}$ have the same number of elements in $\mathrm{K}_{\mathrm{H}_{2}}\left(\mathcal{I}_{n}\right)$.

First we need some notation. Let $1 \leqslant r, s \leqslant n$. We denote by $H_{s}^{\tau}$ the $\mathfrak{H}$-class of $J_{n-1}$ of the elements $x$ such that $\operatorname{Dom}(x)=\{1,2, \ldots, n\} \backslash\{r\}$ and $\operatorname{Im}(x)=\{1,2, \ldots, n\} \backslash\{s\}$. We define $K_{s}^{r}=H_{s}^{r} \cap \mathrm{K}_{\mathrm{H}_{2}}\left(\mathcal{I}_{n}\right)$.

LEMMA 4.17. For $1 \leqslant r, s, u, t \leqslant n$, there exist $\sigma, \nu \in \mathcal{A}_{n}$ such that $\sigma K_{s}^{r} \nu=K_{v}^{u}$ and $\sigma K_{v}^{u} \nu=K_{s}^{r}$.

Now we look at the relations of $\mathcal{I}_{n}$ given in Subsection 3.1. The word cac corresponds to an element of rank $n-2$. Then none of the relations $(c a)^{2}=c a c=(a c)^{2}$ can be applied to an element of rank greater than $n-2$. On the other hand, the words involved in the relations $a^{2}=g^{n}=(g a)^{n-1}=\left(a g^{n-1} a g\right)^{3}=\left(a g^{n-j} a g^{j}\right)^{2}=1$ and $g^{n-1} a g c g^{n-1} a g$ $=$ gacag $^{n-1}=c=c^{2}$ correspond to elements of rank not smaller than $n-1$. Then we have:

LEMMA 4.18. The element of $J_{n-1} \cap \mathcal{I}_{n}$ represented by the word ac does not belong to $\mathrm{K}_{\mathrm{H}_{2}}\left(\mathcal{I}_{n}\right)$.

Proof: By Proposition 1.14, if an element $x \in \mathcal{I}_{n}$ belongs to $\mathrm{K}_{\mathrm{H}_{2}}\left(\mathcal{I}_{n}\right)$, then there exists a word $u$ representing $x$ such that

$$
\begin{cases}|u|_{a} \equiv 0 & \bmod 2 \\ |u|_{g} \equiv 0 & \bmod 2\end{cases}
$$

We shall conclude that no word representing the same element of $\mathcal{I}_{n}$ other than ac satisfies the condition (2), which proves that the element of $\mathcal{I}_{n}$ represented by ac does not belong to $\mathrm{K}_{\mathrm{H}_{k}}\left(\mathcal{I}_{n}\right)$.

We have to take the parity of $n$ into account.

If $n$ is even, then it is easy to check that any word $u$ obtained from $a c$ using the relations above is such that $|u|_{a}$ and $|u|_{g}$ have different parities. Thus $|u|_{a} \bmod 2 \neq|u|_{g}$ mod 2 and so the condition (2) is not satisfied. 
If $n$ is odd, by applying to $a c$ the relations above we only obtain words $u$ such that $|u|_{a}$ is odd. Thus also in this case condition (2) is not satisfied, as required.

Let $H$ be a maximal subgroup of $J_{n-1} \cap \mathcal{I}_{n}$. Then $H$ is isomorphic to $\mathcal{S}_{n-1}$ and its derived subgroup, $H^{\prime}$, being isomorphic to $\mathcal{A}_{n-1}$, has index 2 in $H$. Thus, the only subgroups of $H$ containing $H^{\prime}$ are $H$ and $H^{\prime}$ itself. It follows from Lemma 4.18 there exists an element in the $\mathfrak{J}$-class $J_{n-1}$ not belonging to $\mathrm{K}_{\mathrm{H}_{2}}\left(\mathcal{I}_{n}\right)$. As Lemma 4.17 guaranties that all $\mathfrak{H}$-classes have the same number of elements in $\mathrm{K}_{\mathrm{H}_{2}}\left(\mathcal{I}_{n}\right)$, then each $\mathfrak{H}$-class has precisely half of the elements in $\mathrm{K}_{\mathrm{H}_{2}}\left(\mathcal{I}_{n}\right)$.

We have proved the result announced:

PROPOSITION 4.19. $\mathrm{K}_{\mathrm{H}_{2}}\left(\mathcal{I}_{n}\right)=\mathrm{K}_{\mathrm{Ab}}\left(\mathcal{I}_{n}\right)$. Finally, using Propositions 4.15, 4.19 and 1.9 we get the main result of this subsection:

THEOREM 4.20. Let $\pi$ be a supernatural number and $\mathrm{H}_{\pi}$ the associated pseudovariety of Abelian groups. Then $\mathrm{K}_{\mathrm{H}_{\pi}}\left(\mathcal{I}_{n}\right)=\mathrm{K}_{\mathrm{Ab}}\left(\mathcal{I}_{n}\right)$ if $\pi$ is even and $\mathrm{K}_{\mathrm{H}_{\pi}}\left(\mathcal{I}_{n}\right)=\mathcal{I}_{n}$ if $\pi$ is odd.

\section{Consequences}

The notion of $\mathrm{H}$-kernel is closely related to an important operator of pseudovarieties: the $\mathrm{Mal}^{\prime}$ 'cev product (see [18]). Its definition, when the first factor is a pseudovariety $V$ of monoids and the second factor is a pseudovariety $H$ of groups, may be given as follows:

$$
\mathrm{V} \oplus \mathrm{H}=\left\{M \in \mathrm{M}: \mathrm{K}_{\mathrm{H}}(M) \in \mathrm{V}\right\} \text {. }
$$

Let POI, PODI, POPI and PORI be the pseudovarieties of monoids generated respectively by $\left\{\mathcal{P O} \mathcal{I}_{n} \mid n \in \mathbb{N}\right\},\left\{\mathcal{P O D I} \mathcal{I}_{n} \mid n \in \mathbb{N}\right\},\left\{\mathcal{P O P} \mathcal{I}_{n} \mid n \in \mathbb{N}\right\}$ and $\left\{\mathcal{P O} \mathcal{R I}_{n} \mid n \in \mathbb{N}\right\}$.

In [9, Corollaries 3.7 and 3.8] the second and third authors observed that PODI $\subset$ POI $(9) A b$ and that PORI $\subseteq$ POPI (m) Ab. From the work done here, better bounds can be stated. In fact, using Corollary 4.4 we obtain the following:

COROLlary 5.1. The inclusion PODI $\subseteq$ POI $\cong \mathrm{H}_{2}$ holds.

Similarly, using Corollary 4.12 , we have:

Corollary 5.2. The inclusion PORI $\subseteq$ POPI $\oplus \mathrm{H}_{2}$ holds.

The work presented in this paper was originally motivated by an attempt to compare these pseudovarieties. Although possibly far from obtaining a solution, we leave here the following conjectures:

CONJECTURE 5.3. The equality $\mathrm{PODI}=\mathrm{POI}(\mathrm{m}) \mathrm{H}_{2}$ holds.

CONJECTURE 5.4. The equality $P O R I=P O P I ~ \oplus \mathrm{H}_{2}$ holds. 


\section{REFERENCES}

[1] J. Almeida, Finite semigroups and universal algebra, (English translation) (World Scientific, Singapore, 1995).

[2] J. Almeida and B. Steinberg, 'Iterated semidirect products with applications to complexity', Proc. London Math. Soc. 80 (2000), 50-74.

[3] C.J. Ash, 'Inevitable graphs: a proof of the type II conjecture and some related decision procedures', Internat. J. Algebra Comput. 1 (991), 127-146.

[4] E. Cordeiro and M. Delgado, 'Computing relative abelian kernels of finite monoids', $J$. of Algebra (to appear).

[5] M. Delgado, 'Abelian pointlikes of a monoid', Semigroup Forum 56 (1998), 339-361.

[6] M. Delgado, 'Commutative images of rational languages and the abelian kernel of a monoid', Theor. Inform. Appl. 35 (2001), 419-435.

[7] M. Delgado and V.H. Fernandes, 'Abelian kernels of some monoids of injective partial transformations and an application', Semigroup Forum 61 (2000), 435-452.

[8] M. Delgado and V.H. Fernandes, 'Solvable monoids with commuting idempotents', Internat. J. Algebra Comput. 15 (2005), 547-570.

[9] M. Delgado and V.H. Fernandes, 'Abelian kernels of monoids of order-preserving maps and of some of its extensions', Semigroup Forum 68 (2004), 435-456.

[10] M. Delgado, V.H. Fernandes, S. Margolis and B. Steinberg, 'On semigroups whose idempotent-generated subsemigroup is aperiodic', Internat. J. Algebra Comput. 14 (2004), 655-665.

[11] M. Delgado and J. Morais, 'SgpViz, a GAP [27] package to visualize finite semigroups (http://www.gap-system.org/Packages/sgpviz.html)'.

[12] M.S. Dummit and R.M. Foote, Abstract algebra (Prentice-Hall, Englewood Cliffs, 1991).

[13] V.H. Fernandes, 'Semigroups of order-preserving mappings on a finite chain: a new class of divisors', Semigroup Forum 54 (1997), 230-236.

[14] V.H. Fernandes, 'The monoid of all injective orientation-preserving partial transformations on a finite chain', Comm. Algebra 28 (2000), 3401-3426.

[15] V.H. Fernandes, 'The monoid of all injective order-preserving partial transformations on a finite chain', Semigroup Forum 62 (2001), 178-204.

[16] V.H. Fernandes, 'Presentations for some monoids of partial transformations on a finite chain: a survey', in Semigroups, Algorithms, Automata and Languages, (G.M.S. Gomes, J.-E. Pin and P.V. Silva, Editors) (World Scientific, River Edge, N.J., 2002), pp. 363-378.

[17] V.H. Fernandes, G.M.S. Gomes and M.M. Jesus, 'Presentations for some monoids of injective partial transformations on a finite chain', Southeast Asian Bull. Math. 28 (2004), 903-918.

[18] K. Henckell, S. Margolis, J.-E. Pin and J. Rhodes, 'Ash's type II theorem, profinite topology and Malcev products. Part I', Internat. J. Algebra Comput. 1 (1991), 411-436.

[19] B. Herwig and D. Lascar, 'Extending partial automorphisms and the profinite topology on free groups', Thans. Amer. Math. Soc. 352 (2000), 1985-2021.

[20] J.M. Howie, Fundamentals of semigroup theory, London Mathematical Society Monographs. New Series 12 (Oxford University Press, New York, 1995).

[21] E.H. Moore, 'Concerning the abstract groups of order $k$ ! and $\frac{1}{2} k$ ! holohedrically isomorphic with the symmetric and the alternating substitution groups on $k$ letters', Proc. London Math. Soc. (1) 28 (1897), 357-366. 
[22] L.M. Popova, 'The defining relations of certain semigroups of partial transformations of a finite set', (Russian), Leningradskij gosudarstvennyj pedagogicheskij institut imeni $A$. I. Gerzena, Uchenye Zapiski 218 (1961), 191-212.

[23] J. Rhodes and B. Tilson, 'Improved lower bounds for the complexity of finite semigroups', J. Pure Appl. Algebra 2 (1972), 13-71.

[24] L. Ribes and P.A. Zalesskir, 'On the profinite topology on a free group', Bull. London Math. Soc. 25 (1993), 37-43.

[25] L. Ribes and P.A. Zalesskiŭ, 'The pro-p topology of a free group and algorithmic problems in semigroups', Internat. J. Algebra Comput. 4 (1994), 359-374.

[26] B. Steinberg, 'Monoid kernels and profinite topologies on the free Abelian group', Bull. Austral. Math. Soc. 60 (1999), 391-402.

[27] The GAP Group., 'GAP - groups, algorithms, and programming, Version 4.4', (2004). (http://ww.gap-system.org).

Instituto Politécnico de Bragança

Escola Superior de Tecnologia e Gestão

Campus de Santa Apolónia

5301-857 Bragança

Portugal

e-mail emc@ipb.pt

Departamento de Matemática

Universidade Nova de Lisboa

Monte da Caparica

2829-516 Caparica

Portugal

and

C.A.U.L.

1649-003 Lisboa

Portugal

e-mail: vhf@fct.unl.pt
Departamento de Matemática Pura

Faculdade de Ciências

4169-007 Porto

Portugal

e-mail:quadmdelgado@fc.up.pt 